Techniques \& Culture

Revue semestrielle d'anthropologie des techniques

$59 \mid 2012$

Itinéraires de coquillages

\title{
Murex barclayi : livres de coquilles
}

Murex barclayi: books shells

\section{Claude Sorgeloos}

\section{(2) OpenEdition}

Journals

Édition électronique

URL : https://journals.openedition.org/tc/6594

DOI : $10.4000 /$ tc. 6594

ISSN : 1952-420X

Éditeur

Éditions de l'EHESS

Édition imprimée

Date de publication : 15 décembre 2012

Pagination : 126-149

ISBN : 978-2-7351-1534-1

ISSN : 0248-6016

\section{Référence électronique}

Claude Sorgeloos, "Murex barclayi : livres de coquilles », Techniques \& Culture [En ligne], 59 | 2012, mis en ligne le 15 décembre 2015, consulté le 29 septembre 2022. URL : http://journals.openedition.org/ tc/6594; DOI : https://doi.org/10.4000/tc.6594 


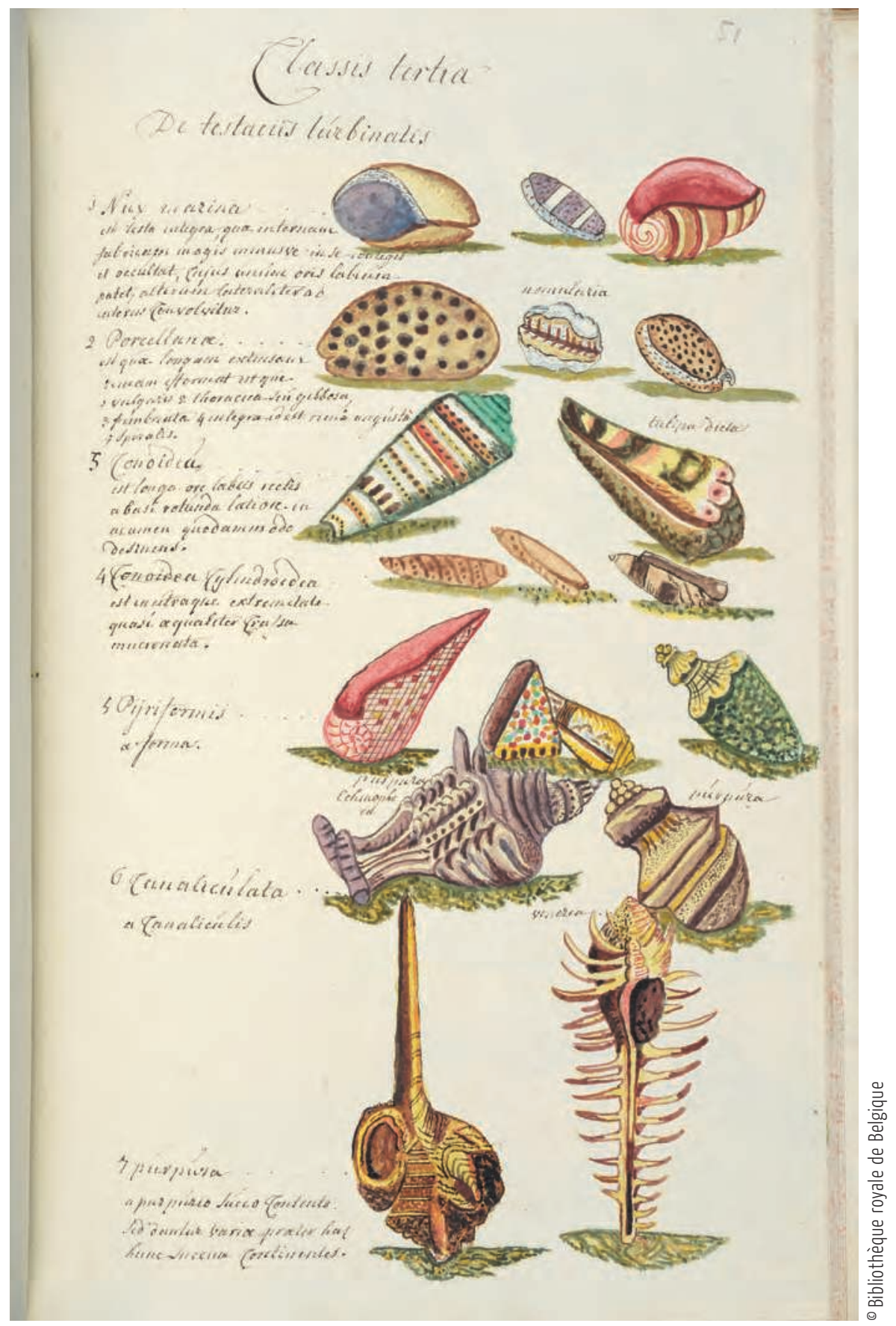




\section{Claude Sorgeloos}

Bibliothèque royale de Belgique claude.sorgeloos@kbr.be
Itinéraires de coquillages - 2

Techniques \& Culture 59, 2012/2 : 126-149

\section{MUREX BARCLAYI : LIVRES DE COQUILLES}

L'intérêt pour les coquillages ne s'est jamais démenti. Traduit dans les livres, il a généré des genres parallèles et complémentaires. Nous envisagerons ici sommairement les genres et formes des livres de coquilles, de manière diachronique et sans refaire cinq siècles de bibliographie sur le sujet, étudié par ailleurs (Nissen 1961, Coppens 1986, Dance 1986, Heppell 1995).

\section{Genres}

Les premières coquilles font leur apparition dans Aristote, Dioscoride et Pline, dans les manuscrits, et les éditions des $\mathrm{XV}^{e}$ et $\mathrm{XVI}^{\mathrm{e}}$ siècles les intègrent dans leur propos. Les traités d'histoire naturelle leur réservent une place parmi les animaux marins, puis les coquilles sont regroupées en chapitres cohérents tandis que se multiplient les espèces et que s'organisent les trois règnes de la nature. L'abondance de spécimens apportés en Europe grâce à la colonisation, au commerce maritime puis aux expéditions scientifiques, donne naissance à deux genres parallèles : les livres de curiosités et les traités de conchyliologie (Eisenstein 1982, Jammes 1984) (Figure 1).

Au XviII ${ }^{e}$ siècle, les collections de curiosités se transforment en cabinets d'histoire naturelle et certains sont à l'origine de musées publics (Visser 2005). Les inventaires se font de manière raisonnée et les coquilles se regroupent en familles en se référant à la systématique, 
Les cabinets de curiosités renfermant des coquilles (Coomans 1992,

Schnapper 1988 : 71-77), éclatant symbole de prestige social, constituent une appropriation de la nature, qui devient signe de culture. Les cabinets engendrent dès lors une forme de littérature qui leur est propre, de savantes publications présentant un choix de curiosités de l'art et de la nature assurant la pérennité du collectionneur ou du prince (Lestringant 2008).

Adam Olearius, Gottorffische Kunst-Cammer, Schlesswig, 1666, cabinet des ducs Frédéric III et Christian-Albert de Schleswig-Gottorp, tabula XXXI, cônes placés horizontalement

(Bibliothèque royale de Belgique, II $12.161 \mathrm{~A})$.

(Fig. 1)

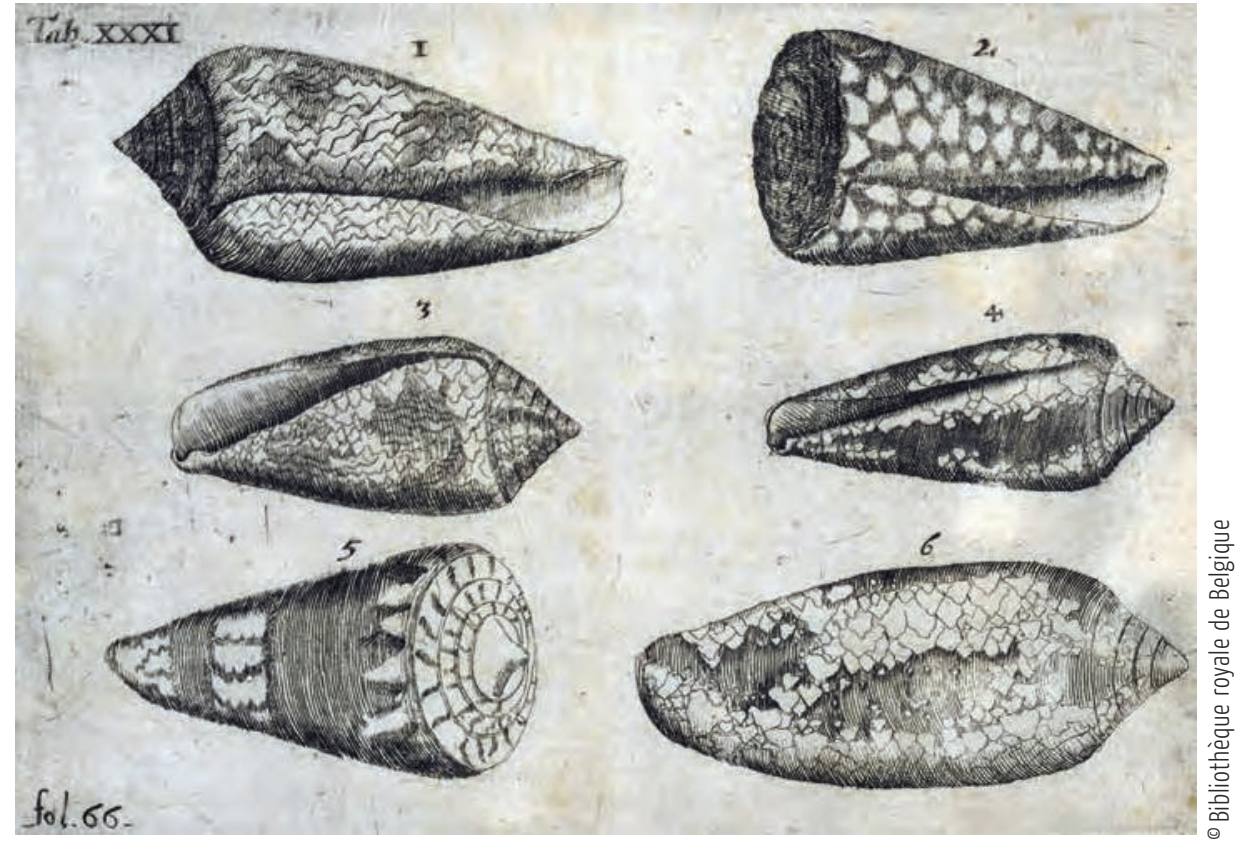

La conchyliologie dérive de l'histoire naturelle, mais elle a des liens étroits avec

les cabinets de curiosités et d'histoire naturelle, tant les naturalistes y puisent l'essentiel de leur connaissance des mollusques.

La Ricreatione dell'Occhio e della Mente (1681) de Philippo Buonanni [9] constitue un livre pionnier en conchyliologie. En France, La conchyliologie de Dezallier d'Argenville éditée en 1742 [19] devient rapidement une référence obligée, comme le Neues systematisches Conchylien-Cabinet (1769-1795) de Martini et Chemnitz [34].

Philippo Buonanni, Recreatio mentis et oculi in observatione animalium testaceorum. Rome, 1684, nos 229-230, Cochlea muricata et Cochlea sarmatica (Bibliothèque royale de Belgique, VH $7.110 \mathrm{~A})$. (Fig. 2)
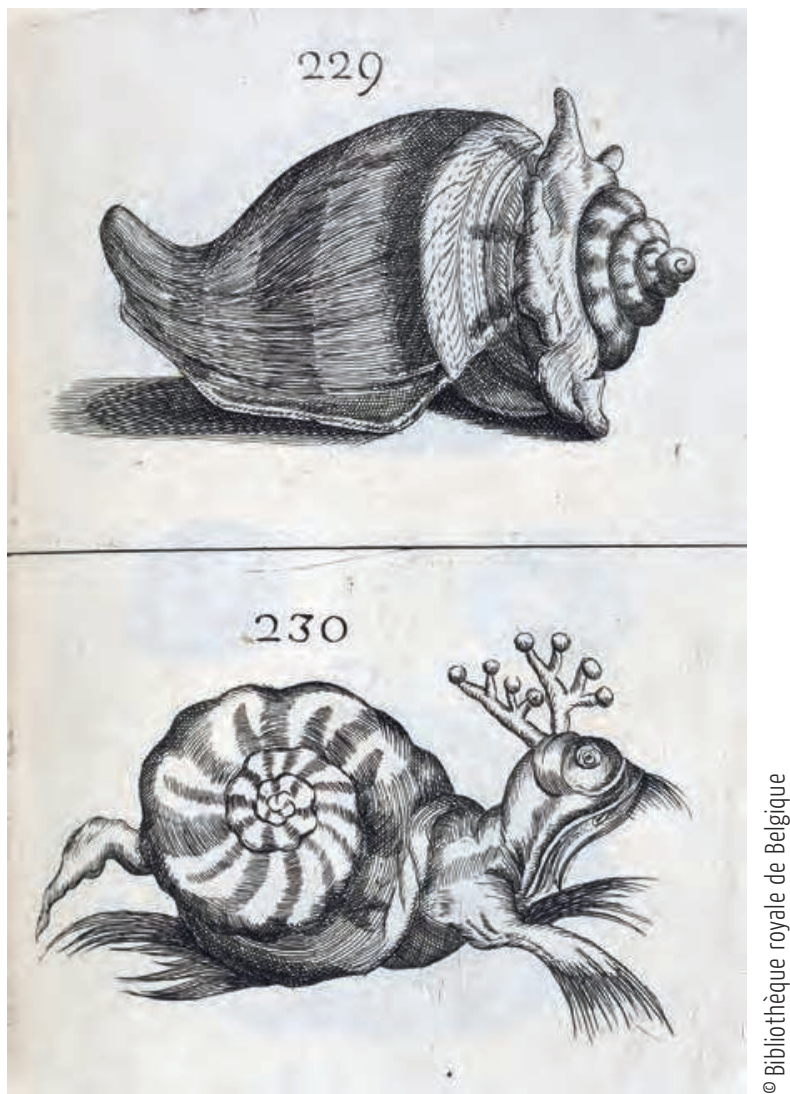
elle-même changeante. Les coquilles changent de statut (Figure 2). Les merveilles de la nature se transforment en spécimens d'histoire naturelle et le nautile monté sur pied de la Renaissance se déplace, étant promu au rang d'espèce de Nautilus. Puis les voyages scientifiques succèdent aux expéditions coloniales et fournissent leur lot de coquilles.

Les traités sur les fossiles constituent un autre genre encore, les coquilles s'offrant au voyage dans le temps. Les fossiles d'ammonites récoltés au sommet des montagnes sont autant de coquilles pétrifiées soulevant le problème du déluge universel et de l'histoire du monde, notamment sur un plan théologique (Figure 3). La science connaît ensuite son évolution propre en devenant géologie, paléontologie et paléobiologie.

La conchyliologie verse progressivement dans la malacologie. À la Renaissance et à l'âge baroque, on ne s'intéresse qu'aux coquilles rares et lointaines, rarement aux parties molles, autrement dit à l'animal complet (Figure 4). Depuis les travaux de Cuvier [17], la malacologie traite des mollusques dans leur totalité. Le terme de malacologie est utilisé par Rafinesque en 1814 [43] et repris par Ducrotay de Blainville en 1825 [20], mais le mot consacre en fait une pratique antérieure (Rafinesque-Schmaltz [43] 1814 : 48, Ducrotay de Blainville [20] 1825: 2, Dance 1986 : 197-200). Cubières étudie leur mode de reproduction en 1800 [16], Fichtel traite des coquilles microscopiques en 1803 [21] (Figure 5). On s'intéresse désormais à l'ensemble des mollusques, d'eau douce et terrestres inclus.

Le développement de la malacologie entraîne un recentrage du contenu. Les naturalistes dressent des inventaires régionaux, traitent de zones terrestres et de régions côtières nationales ou exotiques. Le phénomène colonial est toujours à prendre en compte. Les chercheurs font l'inventaire de leurs colonies. Les mollusques de l'Inde sont répertoriés dans la Conchologia indica de Hanley et Theobald en 1870-1876 [25], deux naturalistes britanniques. L'immensité de l'empire britannique, sa vocation maritime et sa propension à collectionner les îles expliquent en partie l'abondance de la production éditoriale anglo-saxonne, ainsi que le caractère fécond d'une dynastie d'illustrateurs et naturalistes spécialisés, les Sowerby (Coppens 1986 : 101103). Outre les colonies, i y a les zones d'influence : le catalogue des coquilles du Panama est publié, non par un Panaméen, mais par un Américain, C.B. Adams en 1852 [1].

Les coquilles circulent entre collectionneurs par le biais de ventes publiques et de catalogues de curiosités naturelles. Et comme s'il s'agissait d'une mise en abyme, les livres de coquilles sont eux-mêmes collectionnés, cédés ou vendus. Philippe Dautzenberg lègue son impressionnante collection de coquilles et sa bibliothèque à l'Institut royal des Sciences naturelles de Belgique. Antonio Nora à Porto forme une bibliothèque exclusivement consacrée aux coquillages, dispersée à Amsterdam en $1999^{2}$.

Un autre genre né au xix siècle : les guides pour collectionneurs, amateurs et livres de vulgarisation, les collections de coquillages se démocratisant et n'étant plus réservées à une élite. The Voyager's companion de Mawe [36] et The Young Conchologist de Hanley [24] sont destinés à la jeunesse et aux collectionneurs (Dance 1986 : 93-97). Les coquilles font aussi leur apparition dans des livres à usage scolaire, tel l'atlas d'histoire naturelle de Burmeister en 1835 [11]. Les livres sur les usages culinaires des mollusques, quels qu'ils soient, constitueront un autre genre encore (Figure 6).
Scheuzer pose bien le problème des « restes du déluge » et les illustre dans sa Physica sacra (1731) [48] (Scheuchzer [48] (1731), planches 46-60). Ici aussi les liens entre cabinets d'histoire naturelle et amateurs sont très étroits. L'Oryctographie de Bruxelles (1784) de François-Xavier Burtin [12] traite des fossiles du Brabant ; le livre est en grande partie fondé sur sa propre collection.

Georg Wolfgang Knorr, Johann Ernst Immanuel Walch, Die Naturgeschichte der Versteinerungen zur Erläuterung der Knorrischen Sammlung von Merkwürdigkeiten der Natur, II-1, Nuremberg, 1768, tabula la, ammonites (Bibliothèque royale de Belgique, \|I 5.057 C).

(Fig. 3) 


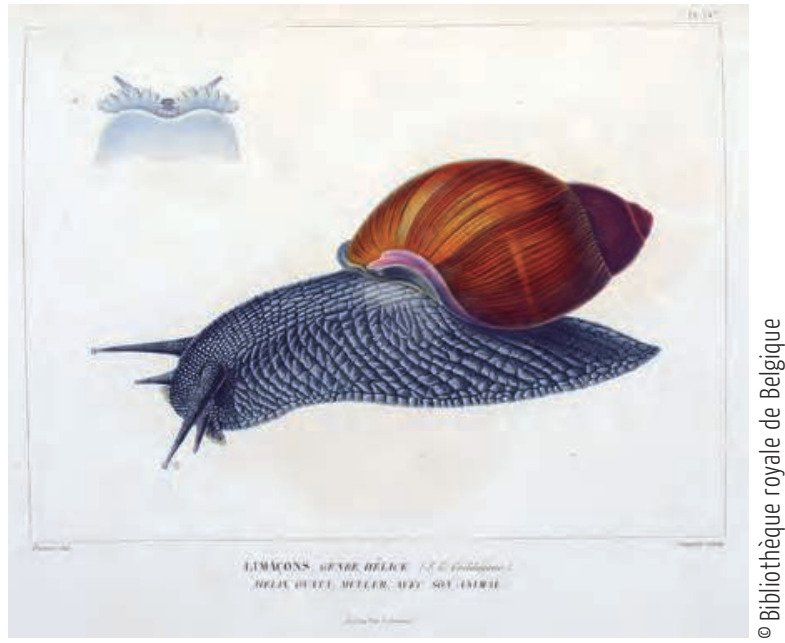

Au xvile siècle, Martin Lister [32] et Valentini [54] procèdent à des dissections de mollusques. Au xvile, on les étudie dans les académies, dont les moules et leurs maladies, à vous ôter « pour toujours l'envie d'en manger » (Marx 1977 : 58).

D. de Férussac, G.-P. Deshayes, Histoire naturelle générale et particulière des mollusques terrestres et fluviatiles. Atlas, II, Paris, 1820-1851, planche 147, Helix ovata ou limaçon (Bibliothèque royale de Belgique, II 29.583 C)

(Fig. 4)

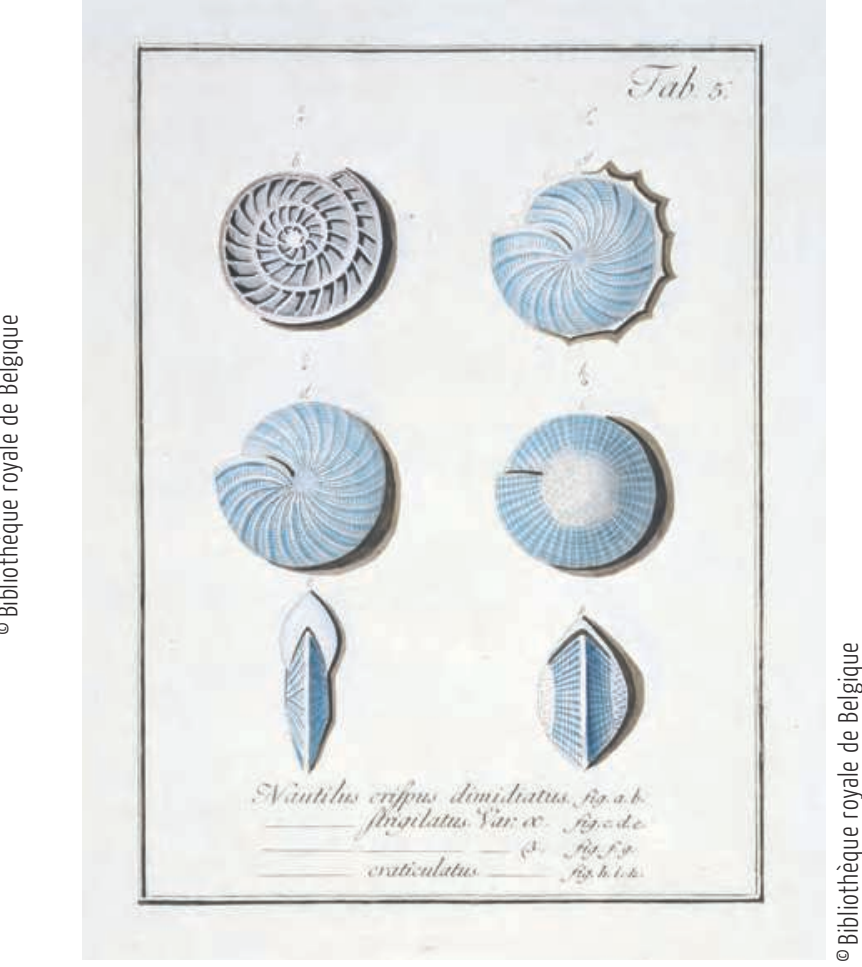

Leopold von Fichtel, Johan Paul Carl von Moll, Testacea microcospica... ex generibus Argonauta et Nautilus.../ Microscopische und andere Schalthiere aus den Geschlechtern Argonaute und Schiffer, Vienne, 1803, planche 5, Nautilus crispus dimidiatus, Nautilus strigilatus, Nautilus craticulatus (Bibliothèque royale de Belgique,

II 29.611 B). (Fig. 5)
Eustace Clare Grenville Murray, The Oyster : where, how and when to find, breed, cook and it eat, Londres, 1861, titre et frontispice (Bibliothèque royale de Belgique, || 29.618 A).

Dans un livre de Murray de 1861 illustré de bois dans l'esprit de Lewis Caroll on traite déjà des préparations de l'huître [39], en forme de caricatures, si tant est que l'on puisse caricaturer une huître (Fig. 6).

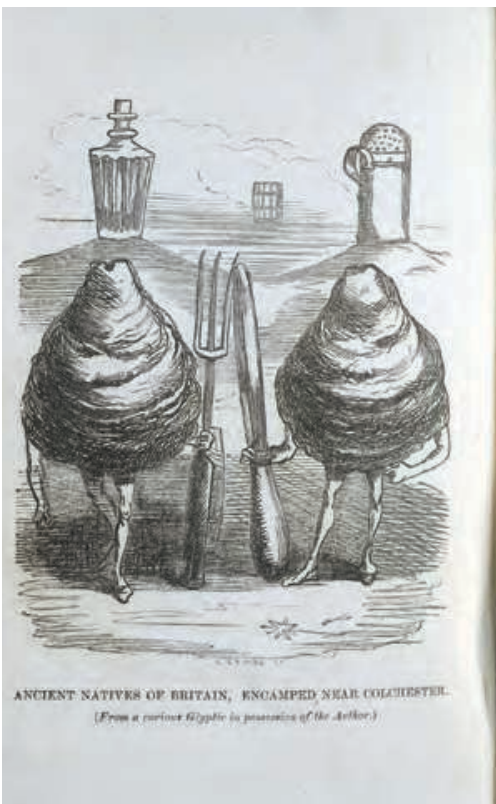

\section{THE OY STER ;} WHERE, HOW, AND WHEN

FIND, BREED, COOK, s.

EAT IT.

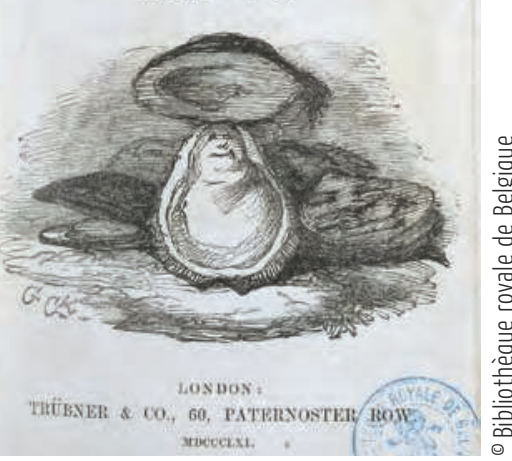




\section{Formes : textes et illustrations}

Les textes se développent et se structurent progressivement en fonction du nombre croissant de coquilles formant les cabinets, tandis que des index multilingues se développent, comprenant les noms latins et vernaculaires. Le catalogue du Muséum de Vienne publié en 1780 par Ignaz von Born [8] en comprend plusieurs : latin, allemand, anglais français et un index belgicus, soit en néerlandais. Le regard du lecteur voyage ainsi du texte aux index et inversement, empruntant des chemins différents en fonction de la langue et de la nationalité du lecteur. Il se déplace aussi vers les illustrations.

S'agissant de livres scientifiques, le propos se doit d'être démontré afin d'identifier la coquille (Laufer 1982, Bassy 1984, Chatelain-Pinon 2000 : 253-267). Les techniques d'illustration sont celles utilisées pour tous les livres : bois, cuivre, qui rend mieux les formes et dessins, puis la lithographie, un procédé qui jalonne tout le xix siècle en raison de sa rapidité et de son aptitude à reproduire les détails. Cette technique sert encore à la Malacologia del Mar Rosso de Issel en 1869 [28], à l'American marine conchology de Tryon en 1873 [53], au catalogue de la collection Hadfield en 1895 [37] et même au compte rendu de la deuxième expédition antarctique française publié par Édouard Lamy en 1911 [31]. La photographie remplacera progressivement toutes ces techniques.

Les livres anciens recourent volontiers au frontispice

(Fumaroli 1994, Chatelain 2000, Sarmant 2000, Deconinck 2012). Ceux conçus pour les livres de curiosités synthétisent l'ensemble des collections, dont on montre une vue des cabinets et tiroirs, animés par des visiteurs, des putti et des allégories des sciences et des arts. Le portrait du collectionneur sert d'introduction récurrente, de personnification et appropriation de la collection (Figure 7). Les livres de conchyliologie adoptent un autre registre. Les frontispices y constituent des images métaphoriques des bienfaits de la mer et du passage de l'eau à la terre (Figures 8 et 9).

Les frontispices font appel à des personnages mythologiques récurrents: Neptune et Vénus. Pour celle-ci, c'est la Vénus anadyomène qui est privilégiée, issue des ondes et posant sur une conque, par analogie avec la coquille, merveille de la nature, belle et rare, dont la déesse ne serait que la métaphore. Le décor est une plage ou une grotte semée de coquilles apportées par les dieux, des tritons ou des nymphes marines, autrement dit une zone intermédiaire entre les fonds marins d'où proviennent les coquillages et les tiroirs des cabinets d'histoire naturelle auxquels ils sont destinés. Ainsi la plage ou la grotte s'impose-elle souvent comme représentation emblématique d'un rituel de passage de la mer à la terre et du mollusque à la coquille.

Certains frontispices intermédiaires adoptent une ornementation plus baroque (Figure 10).

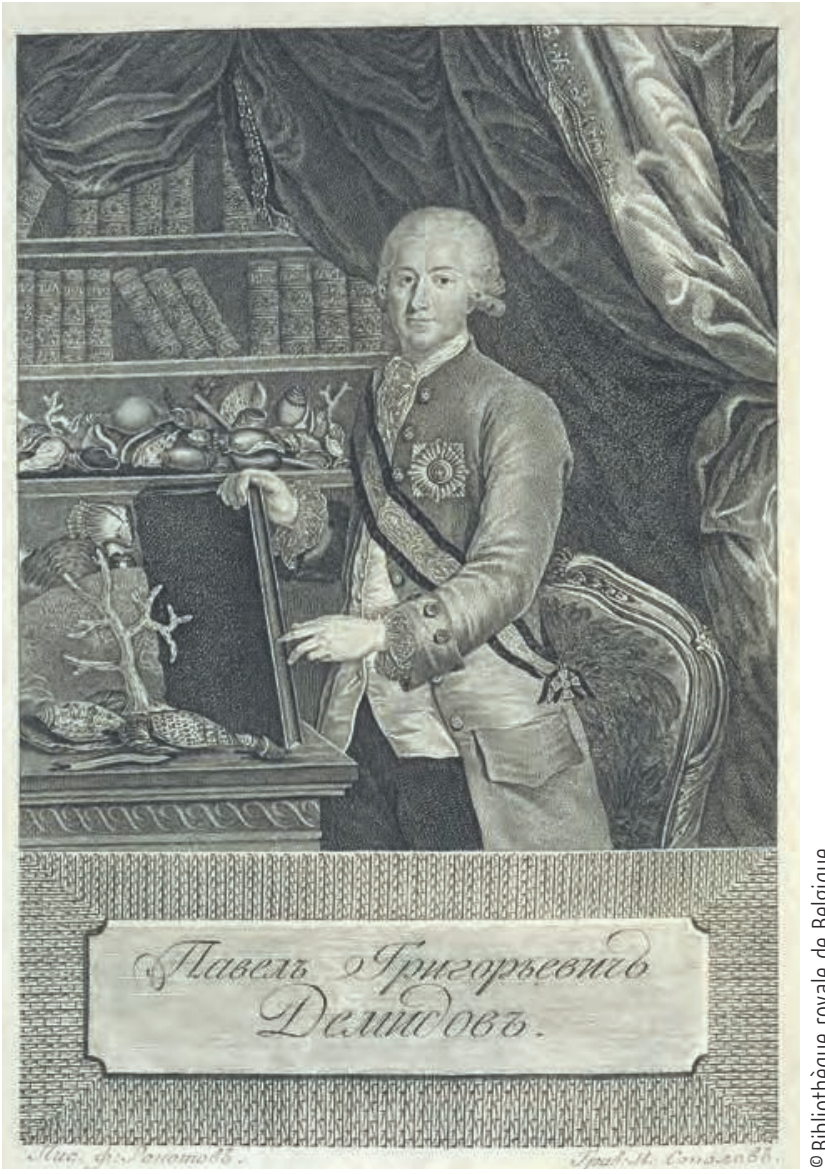

Le plus atypique des portraits de collectionneurs est celui de Georg Everhard Rumphius (1627-1702) : il a la main posée sur ses coquilles, qu'il découvre avec les doigts, étant aveugle [46]. Celui d'Albertus Seba (1665-1736) à Amsterdam, qui a vendu sa collection à Pierre ler de Russie avant d'en commencer une autre, est célèbre [49] (Driessen-van het Reeve 2006). En Russie, en 1804, Paul Demidoff (1738-1821) présente de manière identique au lecteur les coquilles qu'il cédera à l'université impériale de Moscou [18]. Ce modèle de représentation aura une descendance au xixe siècle. Hugh Cuming (1791-1865), grand collectionneur britannique, se fait photographier assis, le bras posé sur une table décorée de coquillages (Dance 1986 : 131 et planche XXV).

Paul Demidoff, Catalogue systématique des livres de la bibliothèque de Paul de Demidoff, I, Moscou, 1806, portrait et coquilles (Bibliothèque royale de Belgique, II 29.509 C). (Fig. 7) 


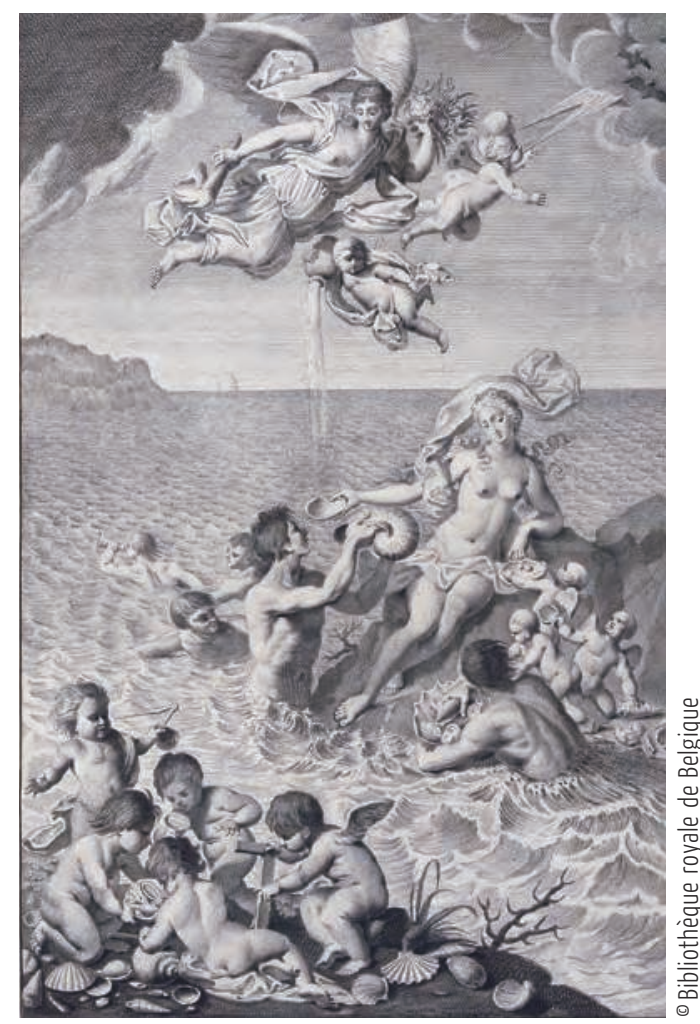

En 1681, le titre gravé de Buonanni montre Neptune sur son char tiré par des chevaux marins, entouré de tritons soufflant dans des conques, tandis qu'un personnage ramasse des coquilles sur la plage. Même décor maritime chez Gualtieri en 1742 [22]. Sur un rocher, Vénus se voit offrir des coquillages, tandis que des putti, à terre, en examinent et en mesurent d’autres.

Niccoló Gualtieri, Index testarum conchyliorum, Florentiae, 1742, frontispice (Bibliothèque royale de Belgique, II 8.021 C). (Fig. 8)

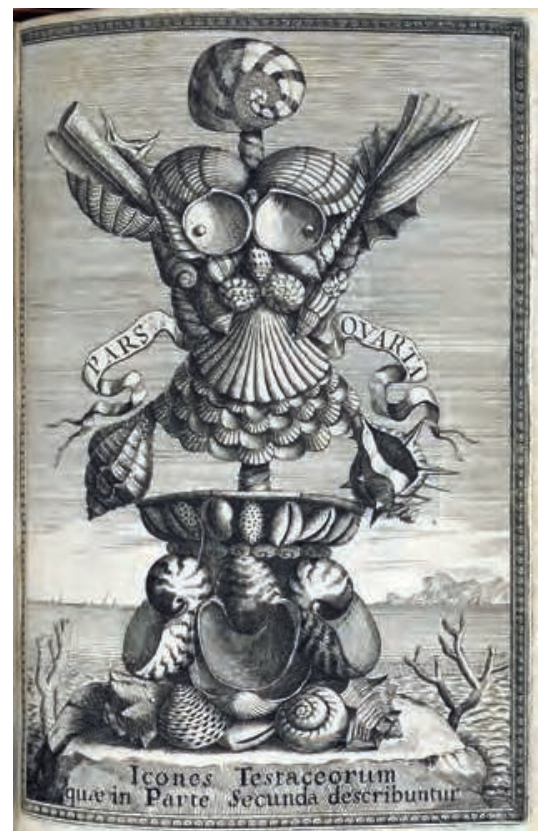

๑ Bibliothèque royale de Belgique

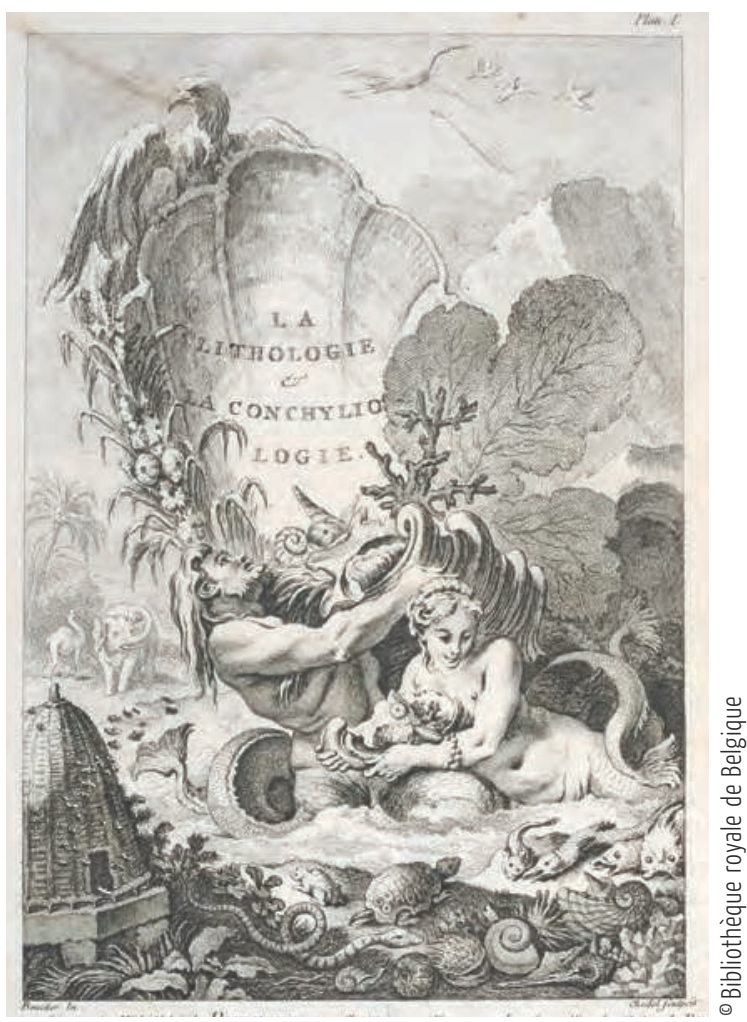

Le titre gravé conçu par François Boucher pour Dezallier d'Argenville offre une coquille géante portant le titre, un triton et sa compagne, des coquillages sur une plage, et d'autres espèces du monde animal, terrestres, marines et volantes, afin de replacer quelque peu le sujet dans l'ordre naturel. En 1764, dans ses Délices des yeux et de l'esprit [29], Knorr reprend le thème de la plage semée de coquilles et d'oursins, tandis qu'une fragile construction de coraux et plantes marines forme un portique ouvert sur la mer, incluant le titre, à l'image des portiques et frontispices introduisant les traités d'histoire et d'archéologie. C'est aussi le parti choisi en 1779 par le graveur de la vignette ornant le titre du traité de Canals y Marti [13] sur la fabrication de la pourpre : Neptune présente des murex tinctoriaux abandonnés sur une plage et ramassés par un berger.

Antoine-Joseph Dezallier d'Argenville, L'Histoire naturelle éclaircie dans deux de ses parties principales, la lithologie et la conchyliologie, Paris, 1742, frontispice de François Boucher (Bibliothèque royale de Belgique, VH 5.804 B).

(Fig. 9)

Les planches de Buonanni sont précédées d'un titre gravé au trophée surmontant une vasque, le tout constitué de coquilles, un monument placé au bord de la mer. Trois cartouches formés de coquilles introduisent en outre les trois classes décrites. L'illustration de Buonanni intègre aussi de remarquables masques faits de coquilles à la manière d'Arcimboldo, qui ne sont là que pour l'ornementation.

Philippo Buonanni, Recreatio mentis et oculi in observatione animalium testaceorum. Romae, 1684, trophée de coquilles. (Bibliothèque royale de Belgique, VH 7.110 A).

(Fig. 10) 


\section{Vignettes, bandeaux et culs-de-lampe}

Destinées aux savants mais aussi aux collectionneurs, les livres de coquilles constituent souvent des publications de luxe. Ils comprennent des ornements spéciaux : bandeaux, culs-de-lampe, vignettes et lettres initiales à décor allusif spécialement conçus pour l'édition. Dans Gualtieri, la vignette ornant les titres et sous-titres est une scène de pêcheurs débarquant des coquillages et on conçoit des lettres initiales à décor de madrépores et de coquilles. Le texte de Rumphius s'ouvre par un bandeau à décor de grotte marine ; le décor s'articule autour de statues de Vénus et Neptune, tandis qu'une allégorie déverse des coquilles d'une corne d'abondance ; au centre, un médaillon représentant un argonaute trône littéralement sur un piédestal.

Ignaz von Born publie à Vienne le catalogue des coquilles rassemblées par l'Empereur François $\mathrm{I}^{\text {er }}$ Étienne. En deux versions, l'une de format in-octavo en 1778 [7], comprenant une seule planche en couleurs, l'autre en 1780 [8] de format in-folio illustrée de 18 grandes planches mises en couleurs alignant soigneusement les coquilles, comme dans les tiroirs d’un muséum. Le texte est en outre agrémenté de bandeaux et culs-de-lampe allusifs, soit 44 vignettes décoratives ou didactiques. La vignette introductive représente des putti rangeant des coquilles dans un cabinet, surmonté des portraits de l'Impératrice Marie-Thérèse d'Autriche et de son époux François $\mathrm{I}^{\mathrm{er}}$, et Neptune tendant une coquille à une allégorie désignant le portrait de l'Empereur, le fondateur de la collection. Rituel de passage encore, mais on omet la plage pour passer directement au cabinet et au muséum. Les autres gravures présentent des coquilles mises en scène, toujours dans un encadrement, telles des tableaux. Le registre verse aussi dans l'hommage rendu aux naturalistes Pline, Aldrovandi, Linné, Chemnitz, Adanson, Davila, Gualtieri, Martini, dont les cénotaphes, placés au bord de la mer, sont honorés par des tritons et sirènes déposant des coquilles au pied des monuments. En cette fin du xvIII siècle, ce livre est caractéristique du statut toujours ambigu des coquilles, à la fois raretés chères aux collectionneurs et spécimens d'histoire naturelle, selon que l'on regarde les vignettes ou les planches. Le xIx ${ }^{e}$ siècle abandonnera les ornements spéciaux.

\section{Apex : le sens des coquilles}

Les planches constituent l'essentiel de l'illustration, mais les images changent progressivement de place et la mise en page évolue en parallèle. Les gravures sur bois et sur cuivre sont insérées dans le texte et le regard passe insensiblement du texte à l'image et de l'image au texte. Mais les espèces se multipliant, les cuivres génèrent de grandes planches à pleine page, souvent hors texte, permettant aux coquilles de déployer leur diversité. Le regard se déplace dès lors du texte aux planches et se développent de nécessaires systèmes de référence, des renvois visuels, des légendes, une numérotation des planches et des coquilles correspondant au texte. Dans certains ouvrages, l'illustration occupe une place au moins égale au texte qui figure en regard. Elle lui est même supérieure car le lecteur découvre d'abord la planche à droite, en tournant la page, avant de lire le texte à gauche. C'est encore le cas, souvent, dans les guides contemporains destinés aux collectionneurs. 
La manière de représenter les spécimens a évolué. Les coquilles sont accompagnées d'une ombre donnant du relief et de la profondeur au sujet. Mais ce n'est pas le seul motif. Elles paraissent ainsi posées sur le sable, procédé dont s'inspireront les frontispices. Dans les livres de curiosités, les coquillages dessinent volontiers des motifs baroques (Figure 11). Les pièces elles-mêmes changent de sens sur les planches. Les univalves sont placés l'apex en bas ou en haut, selon les auteurs et les illustrateurs (Figure 12). On passera progressivement à la position verticale et l'apex sera placé en haut, les coquilles ayant en outre tendance à s'aligner en passant du statut de curiosité à celui d'espèce du monde vivant dont on fait l'inventaire sous la forme de séries, comme dans les tiroirs des musées d'histoire naturelle (Figure 13). Quant aux bivalves, ils sont représentés horizontalement avec leurs deux valves (Figure 14), mais l'usage s'installera de n'en présenter qu'une seule, verticalement, par économie de moyens, afin d'en placer le plus par page. Pour certaines espèces, dont une valve est plus bombée que l'autre, on présente les deux valves réunies et fermées, de profil (Figure 15).

Le catalogue de la collection Seba [49] inclut de fragiles constructions décoratives en forme d'oiseaux ou de masque, en fait à l'image des coquilles rangées dans les tiroirs. Le titre gravé du Wondertooneel der nature publié par Levinus Vincent en 1706 [56] est une vue générale du cabinet, tandis que des planches dépliantes présentent des meubles et tiroirs de coquilles arrangés de manière baroque. Les cabinets hollandais ayant survécu se présentent encore de la sorte (Coomans 1992 : 196-197, fig. 163-165 ; Bergvelt, Meijers, Rijnders 2005 : 179-202)

Albertus Seba. Locupletissimi rerum naturalium thesauri accurata descriptio, III, Amsterdam, 1758, tabula XXXVI, deuX tiroirs de coquilles à présentation baroque, dessinant des motifs d'oiseaux, de corbeilles, d'arcs,

d'étoiles et d'entrelacs (Bibliothèque royale de Belgique, II 8.226 E RP).

(Fig. 11)

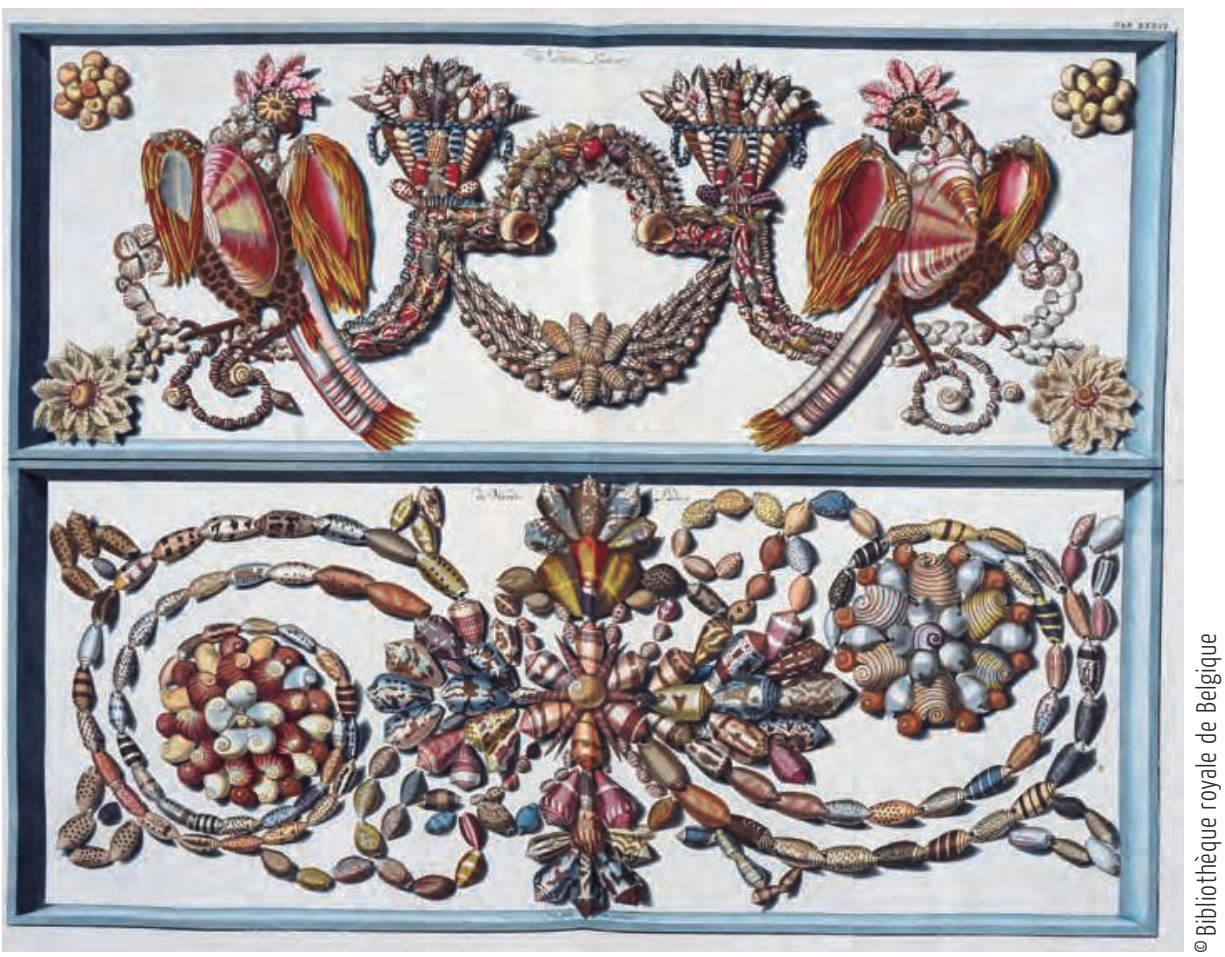

Les coquilles ont aussi un sens naturel, à de rares anomalies près. Pendant la croissance de l'animal, les tours des univalves se déroulent de gauche à droite dans le sens des aiguilles d'une montre à partir de l'apex et ils se terminent sur l'ouverture, à droite. Certains illustrateurs vont toutefois omettre d'inverser le dessin gravé et dès lors imprimer l'illustration dans le mauvais sens. Le résultat imprimé est une coquille inversée à spire de la droite vers la gauche et ouverture à gauche (Dance 1986 : 17). En 1551-1555, 


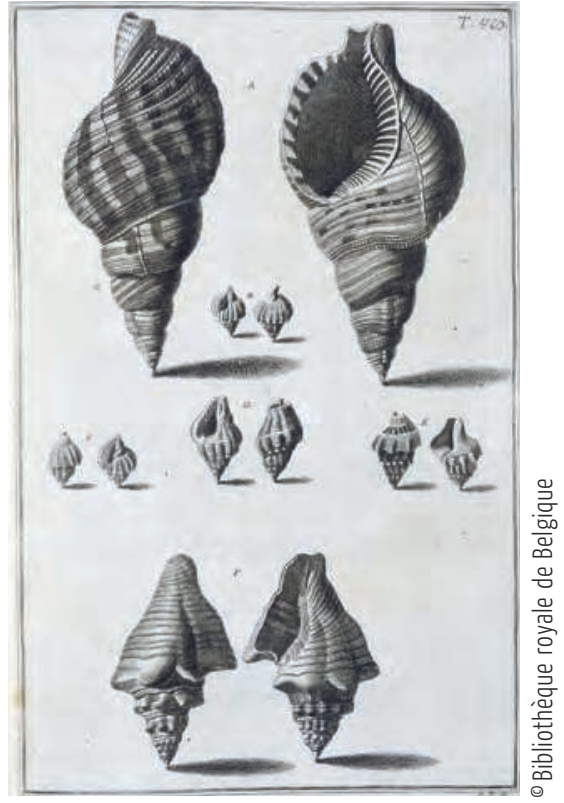

Ces coquillages adoptent la position horizontale dans Rumphius [46]. Gualtieri est assez atypique à cet égard. Les coquilles défient toutes les lois de la physique en étant posées sur la pointe, apex en bas, comme des toupies. Sur les planches de François Valentijn en 1754 [55], les coquilles sont placées en oblique et apex vers le bas.

Niccoló Gualtieri, Index testarum conchyliorum, Florence, 1742 , planche 48 , coquilles posées verticalement sur leur pointe (Bibliothèque royale de Belgique, II 8.021 C). (Fig. 12)

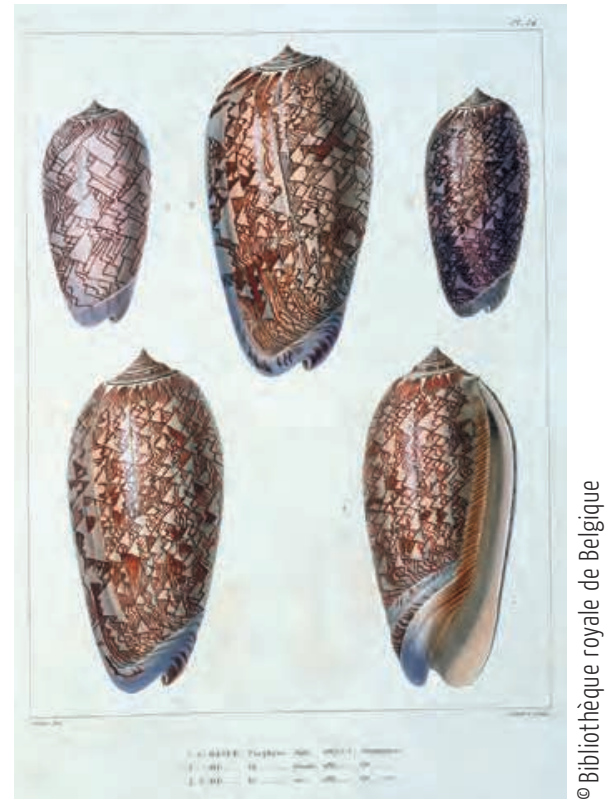

P.L. Duclos, Histoire naturelle générale et particulière de tous les genres de coquilles univalves marines à l'état vivant et fossile, publiée par monographies. Genre olive. Paris, 1835, planche 24 , olives alignées verticalement, apex vers le haut (Bibliothèque royale de Belgique, II 28.017 C). (Fig. 13)
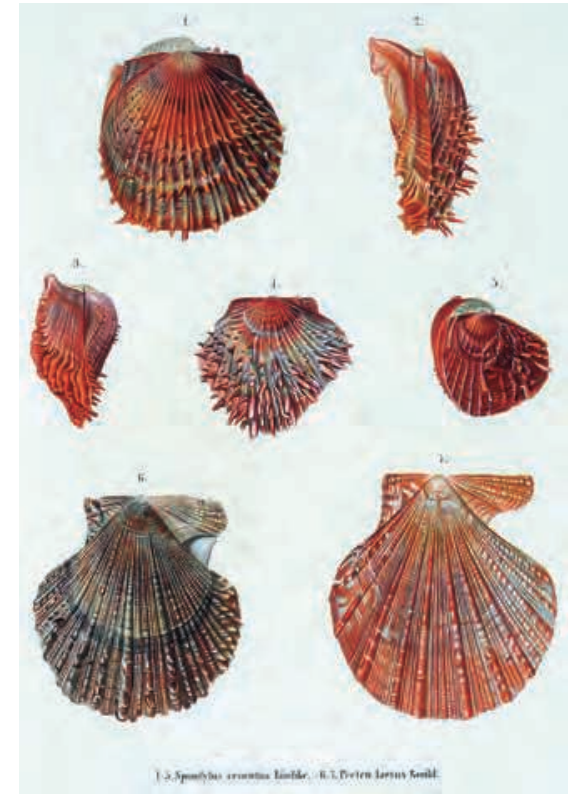

C.E. Lischke, Japanische Meeres-Conchylien, Cassel, 1869, planche XII, spondyles et pectens, une seule valve placée verticalement et deux valves de profil (Bibliothèque royale de Belgique, || 29.682 B)

(Fig. 15) valves (Bibliothèque royale de Belgique, II $8.226 \mathrm{E})$.

(Fig. 14) 


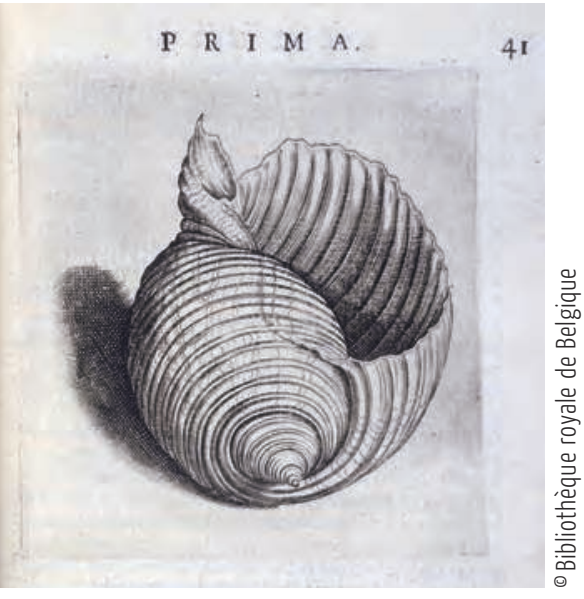

Benedicto Ceruto et Andrea Chiocco, Musaeum Francesc. Calceolari, Vérone, 1622, p. 41,

Tonna galea, sens de la coquille inversé (Bibliothèque royale de Belgique, VB 4.032 (). (Fig. 16)

Pierre Belon, De aquatilibus libri duo, Paris, 1553, p. 380, bois, argonaute voguant sur la mer (Bibliothèque royale de Belgique, VH 7.044 A). (Fig. 17)
l'Historia naturalis d'Adam Lonicerus [33] offre quelques coquillages inversés de ce type et l'erreur se répétera dans l'Historia naturale de Ferrante Imperato en 1599 [27], le Museum F. Calceolarii en 1622 [14] (Figure 16) et d'autres traités. Même Rembrandt commet l'erreur en 1650 en gravant un spécimen de Conus marmoreus, alors que sa signature est parfaitement lisible, donc inversée sur le cuivre avant impression (Coomans 1992 : 202-203). Techniquement, cela ne pose pas de problème et la seule explication est la méconnaissance d'une loi naturelle dans le chef de l'illustrateur. Le phénomène est tout à fait singulier car le problème ne se pose pas pour d'autres espèces, qui ont une tête et des membres, une fleur et des racines.

\section{Un voyage intérieur : le rêve et la spirale d'or}

Il y a aussi les illustrations "rêvées ». Comme le poisson-moine ou le poisson-évêque, le mollusque géant et fabuleux Cochlea sarmatica illustré dans La cosmographie universelle d'André Thévet en 1575 [52] réapparaît dans d'autres traités, dont celui de Buonanni en 1681 et à nouveau dans son Museum Kircherianum de 1709 [10] (fig. 2) (Buonanni [9] 1684 : 142-143 et vignette 230, Johnson 2002). L'image d'un céphalopode argonaute déployant sa membrane et flottant sur les ondes n'a lui aussi cessé d'intriguer depuis Aristote (Pastoureau 1982) (Figure 17). Dans un autre registre, il y a les faux destinés aux collectionneurs et quelquefois reproduits de bonne foi par des naturalistes, telle la porcelaine «souris tachetée » publiée par Favanne fils, en fait une simple Cypraea lurida falsifiée pour en accentuer la rareté (Dance 1986 : 54-55 et fig. 11).

Depuis l'origine, les naturalistes et collectionneurs sont fascinés par les nautiles et argonautes, des pièces rares. La régularité de leurs spires évoque irrésistiblement la spirale d'or. D'où les représentations gravées de nautiles sciés en deux, puis d'autres coquilles, et de spirales schématiquement gravées sur bois, apparaissant dans les traités. Jacques Bernouilli y consacre des travaux [5], puis d'autres. Gualtieri insère régulièrement des

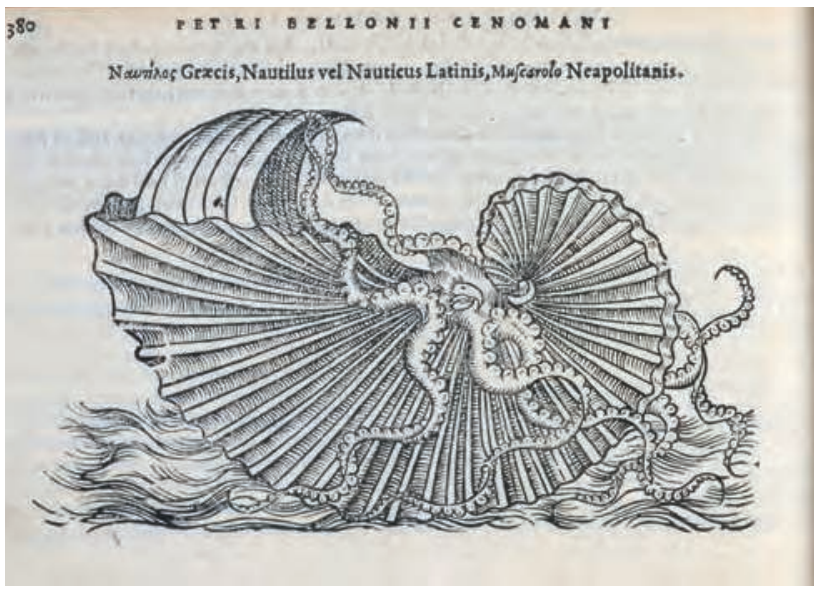

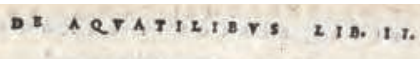

NAVTEVBALTER.

Secundam Nautilis conchx fpeciem ab Ariftocele traditam effe hanc puto, quam noftriartifices in magno pretio ad vafa ditioribus abacis accommoda conficienda habere folent. Pooftriartifices id vocant, quodd Muricis conchx (de qua in Buccino differemus) formam holent . Porcellanam ob Porcellanam nominát, ex qua antiquis vafa qux murrhina dicebantur, fieri folcbat. Eam enim etram

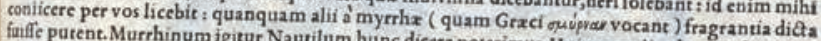
conuenit, fed paulòm magis eft craffas: prior preterea peetunculorum Huius amplitudocum fuperiore flaber, parte interna infinita habens incerftiter , peatunculorum more ftriatus eft : hic omnino iplendens: fubinde cirsunagentabens interftitia : vndecun que nitidius, ac mira colorum varietate mé cerner. 
coquilles sciées en deux sur ses planches, tant leur ouverture invite à pénétrer dans la demeure d'un animal disparu et à effectuer un voyage intérieur. Depuis, la publication de radiographies permet de découvrir l'architecture intérieure des coquilles. Et plus récemment, modélisations et calculs mathématiques contribuent à étudier les coquilles, les mathématiciens ayant investi ce domaine, fascinés par les volutes et courbes adoptées par la nature, en rapport avec la spirale d'or et sa parenté avec la croissance des nautiles ${ }^{3}$.

\section{Stratégies éditoriales}

Sous l'Ancien Régime, les éditeurs, voire quelques auteurs, utilisent des stratégies éditoriales particulières en ciblant le milieu des collectionneurs, le principal public de leurs ouvrages, afin de couvrir une partie des frais. Certains dédient chaque planche à un amateur, qui se transforme dès lors en mécène. Dans son Histoire naturelle du Sénégal, Michel Adanson [2] réserve une large place aux planches de coquilles, après avoir suscité le mécénat de collectionneurs. Dezallier d'Argenville dédie les planches de sa Conchyliologie à des collectionneurs, d'autant que le texte consacre des chapitres importants à la constitution d'un cabinet, à la manière de préparer et conserver des coquilles. Les planches de Georg Wolfgang Knorr et Johann Ernst Immanuel Walch en 1777-1778 [30], sur les catastrophes du globe, représentent des fossiles peints d'après nature extraits des principales collections allemandes et le nom du collectionneur ou du cabinet est gravé en bonne place pour la pérennité. En Hollande, les collectionneurs affectionnent que l'on reproduise leurs coquilles et des annonces paraissent à cet effet (Landwehr 1976 : 87-88). Les livres incluent aussi des listes de collectionneurs, telle la réédition de Dezallier d'Argenville par les Favanne. On vise aussi ce milieu par la souscription. La liste des souscripteurs présente dans le traité des fossiles de Burtin n'est par exemple, qu'une longue liste de collectionneurs passant à la postérité.

\section{Cypraea lurida ou pulchra : le problème de la couleur}

La transition du manuscrit à l'imprimé traduit le passage de l'unique au multiple. Toutefois, les traités relatifs aux coquilles connaissent les problèmes rencontrés par d'autres livres illustrés supportant difficilement cette transition, tout particulièrement les travaux d'histoire naturelle : la couleur ${ }^{4}$. Comment reproduire les couleurs de certaines espèces de porcelaines, d'olives ou de bulles, que ne distinguent que leurs subtiles nuances de coloris. Comment reproduire l'orange doré de Cypraea aurantium ou faire la distinction entre Cypraea lurida et Cypraea pulchra, caractérisées par des tons plus clairs ou plus sombres. Les techniques d'impression des illustrations ne le permettent pas. Les amateurs font donc colorier leurs plus beaux livres de coquilles. Au xviII siècle, les éditeurs et libraires proposent aussi à la vente deux versions : une en noir et blanc et une autre, plus luxueuse et nécessairement plus chère, coloriée à la main. À Bruxelles, la Conchyliologie de Dezallier 
Entre 1640 et 1660, à Milan, Manfredo Settala fait peindre les objets remarquables de sa collection, quelque 300 dessins d'histoire naturelle de toute beauté regroupés en sept volumes. Les coquilles offertes par le grand-duc de Toscane Cosme III de Médicis à Jacopo Cospi à Bologne sont reproduites dans un manuscrit peint ${ }^{6}$ (Conihout 1996 : $294 n^{\circ} 64$ et 65). En 1753, à Bruxelles, le prince Charles de Lorraine fait peindre ses spécimens dans un manuscrit intitulé Plantes marines et coquilles du cabinet de S.A.R., peintes en miniature (Sorgeloos 1996 : 152).

En 1776, Louis-Athanase de Crillon rédige une Description de son cabinet, illustrée de dessins en couleurs présentant un choix de ses coquilles et madrépores les plus rares ou les plus beaux. Ce manuscrit était réservé à un usage particulier : il servait de catalogue de vente que l'abbé faisait circuler parmi les amateurs.

Description très concise du Cabinet de M. l'abbé Crillon, manuscrit, feuille 28 , « concha exotica » et « harpe à côtes serrées». (Institut royal des Sciences naturelles, Bruxelles, Bibliothèque Philippe Dautzen-

berg, 51624).

(Fig. 18)

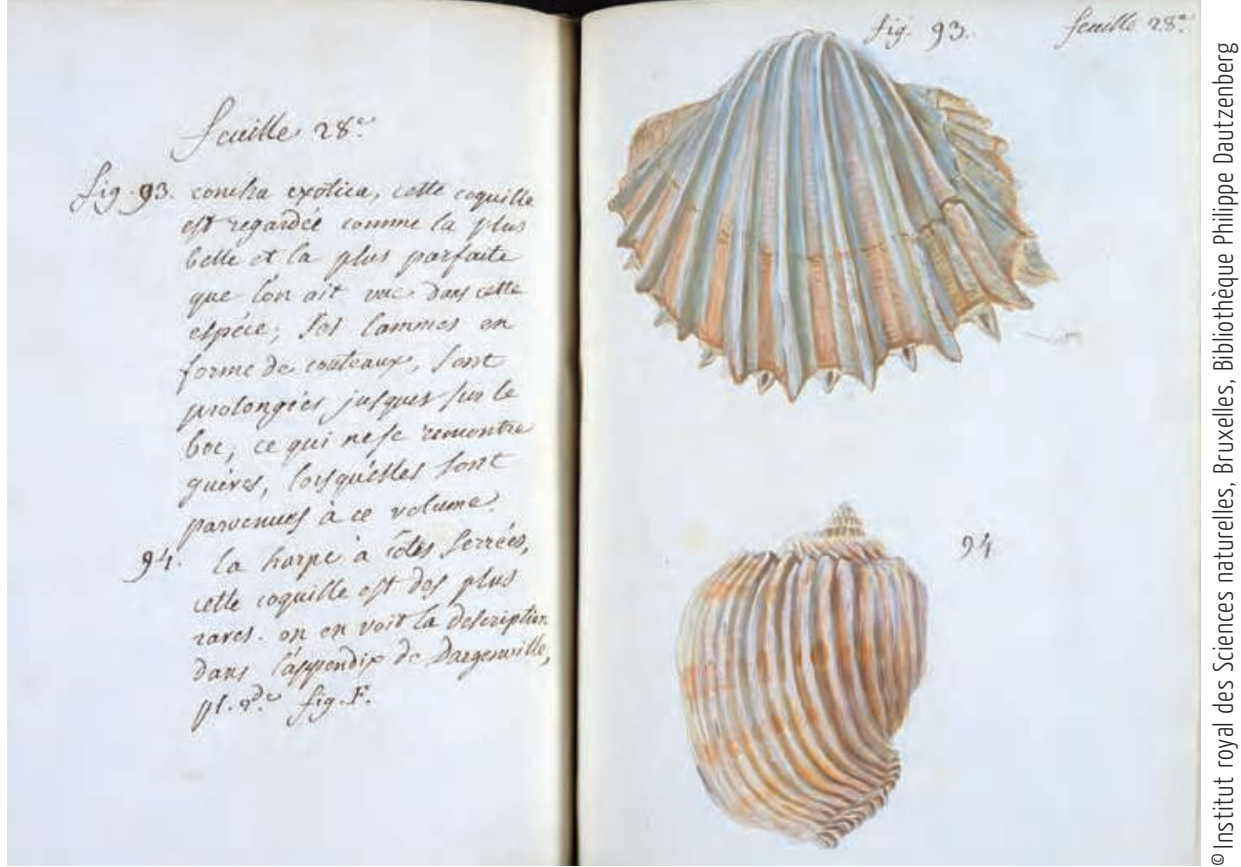

d'Argenville est proposée en noir et blanc au prix de 48 livres, ou en couleurs « d'après nature » pour 140 livres. En 1779, le libraire bruxellois Benoît Lefrancq vend les Centuries de P.J. Buchoz en couleurs ou en noir (Sorgeloos 1996 : 151). En 1829, l'éditeur parisien Roret annonce deux versions de son Atlas des mollusques, avec figures en noir au prix de 7 francs et en couleurs pour 14 francs $^{5}$. Mais le procédé est cher et quelques entreprises éditoriales sont abandonnées: Polydore Roux publie sa première et unique livraison de sa superbe Illustration conchyliologique en 1828 [45], illustrée de 9 lithographies mises en couleurs avec rehauts de gomme, sur les 400 prévues.

Une autre solution est de peindre les coquilles d'après nature (Figure 18), comme en témoignent les vélins du Cabinet du Roi ou d'autres ouvrages ${ }^{7}$ (Lamy 1930 : 17). On découvre parfois dans les manuscrits les hésitations du siècle sur le sens des coquilles (Figure 19: voir photo d'ouverture). Celles-ci servent quelquefois à rehausser d'autres manuscrits, comme le Florilège de Nassau-Idstein de Johann Walters au xviI ${ }^{e}$ siècle, dont les gouaches sur vélin présentent quelquefois des strombes et harpes afin de davantage souligner la beauté des fleurs d'oranger et de lin peintes par l'artiste (Pinault Sørensen 2008 : 105, fig. 37).

$\mathrm{Au}$ xIXe siècle, la chromolithographie permet de rendre l'infinie variété des couleurs offertes par les coquilles, distinguant enfin correctement les espèces entre elles. Avec l'avènement de la photographie, technique nouvelle, le problème des couleurs n'est pas résolu immédiatement. En 1863, Hanley publie Photographic conchology [23] illustrée de 7 planches de photographies, mais mises en couleurs à la main. La chromolithographie reproduira en fait les coquilles jusqu'à l'apparition de la quadrichromie. La part de la couleur est même tellement déterminante que dans les Illustrations conchyliologiques de Chenu [15], le nom du coloriste Gérard figure sur les planches aux côtés des noms des dessinateurs et graveurs (Figure 20). 


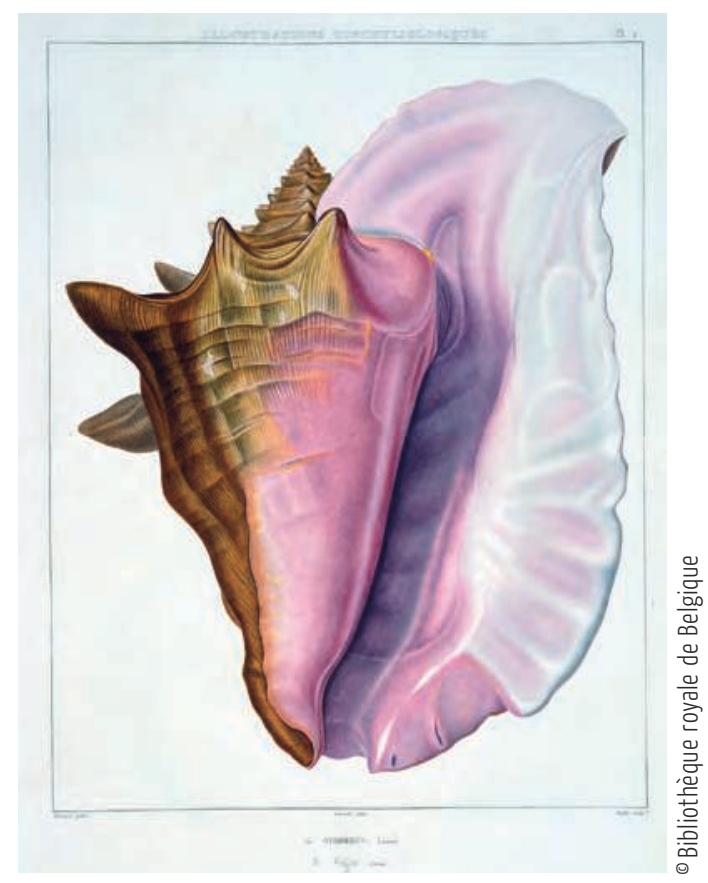

Jean-Charles Chenu, Illustrations

conchyliologiques, I, Paris, 1843,

Strombus Gigas, lithographie, couleurs

du coloriste Gérard

(Bibliothèque royale de Belgique,

II $68.911 \mathrm{E})$.

(Fig. 20)

\section{Formats}

Les livres de coquilles adoptent l'in-folio et l'in-quarto, en raison du caractère universel de la nature, du nombre d'espèces et d'illustrations, puis les formats se réduisent parallèlement à la démocratisation du lectorat. Dans certains cas, on utilise un format oblong, tel Pierre Belon dans son livre sur les poissons en 1553 : les coquilles y dépendent encore des poissons et le but était de placer ceux-ci horizontalement et non verticalement comme dans les grands formats d'ichtyologie habituels (fig. 17). Proche de celui du tableau et des recueils d'estampes, le format oblong permet surtout de peindre la nature. En 1666, Adam Olearius utilise un tel format pour son livre sur les raretés acquises par les ducs Frédéric III et Christian-Albert de SchleswigGottorp [41] (fig. 1). Au XviII siècle, les quatre rares volumes de coquilles édités par Thomas Martyn [35] se présentent eux aussi en format oblong car il s'agit de peindre les merveilles de la mer (Figure 21). Les planches y prennent place sur un recto et le verso en regard est totalement vierge. Les légendes sont reprises dans une liste à la fin et le livre traduit ainsi la prééminence totale de l'image sur le texte, quasi absent, à l'exception d'une introduction. On en trouve des échos modernes, tel le beau recueil de planches en couleurs publié par Yoichiro Hirase en 1914-1915, Kaisenshu [26] : le format plus haut que large ne sort pas des normes, mais les planches doubles se déploient ensuite pour former des tableaux.

Le public n'étant plus le même que les amateurs fortunés, les petits formats sont volontiers utilisés au XIX ${ }^{e}$ siècle pour les guides destinés aux collectionneurs et amateurs, illustrés de simples bois dans le texte, comme The Young Conchologist 
Thomas Martyn The Universal Conchologist. Le Conchologiste Universel, Londres, 1785, figure 59,

Cypraea Aurantium

(Bibliothèque royale de Belgique,

|| 29.589 ()

(Fig. 21)
Le catalogue du cabinet formé par le docteur Joachim Friedrich Bolten à Hambourg en 1819 [40] est illustré de 4 lithographies de spécimens remarquables. G.B. Sowerby dresse celui de la collection du comte de Tankerville en 1825 [50] et le fait suivre de six planches en couleurs, dont un rare Conus gloria maris.

G.B. Sowerby, A catalogue of the shells contained in the collection of the late Earl of Tankerville, Londres, 1825, planche 8 , Conus gloria maris (Bibliothèque royale de Belgique, II $29.601 \mathrm{~A})$. (Fig. 22)

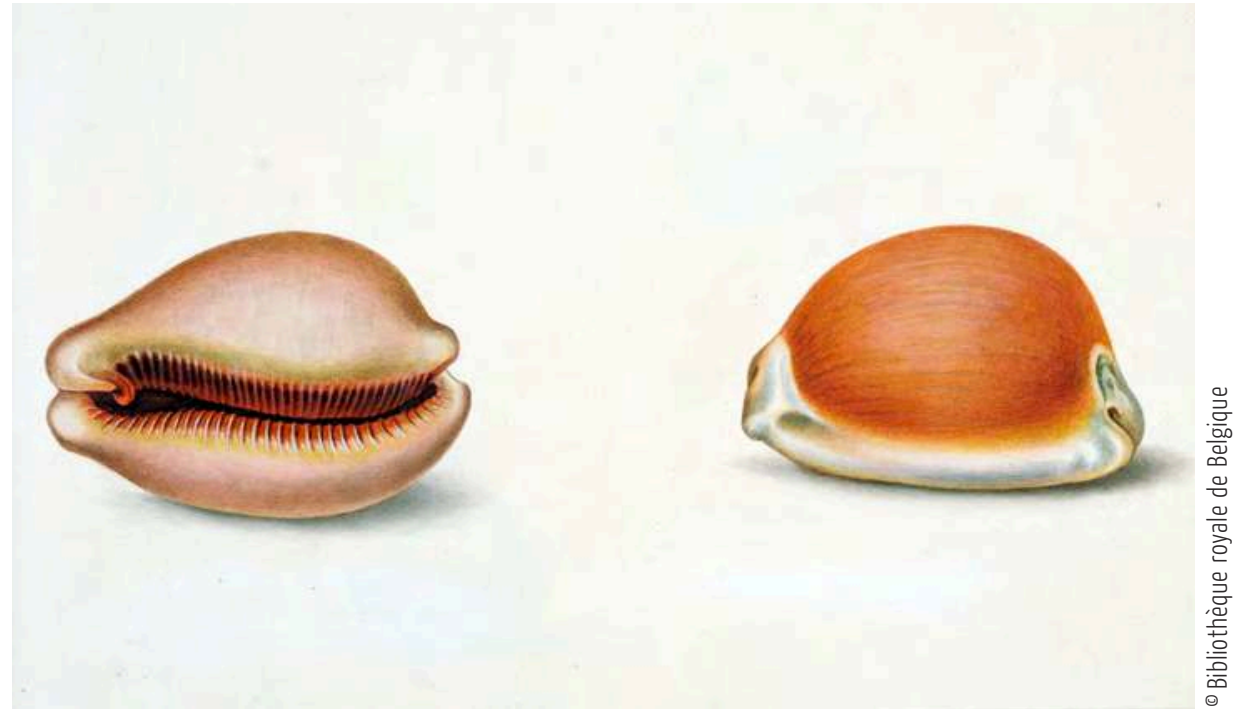

de Hanley [24]. Les ventes de coquilles suscitent des catalogues de petit format, illustrés, et ceci est précoce parmi les catalogues de ventes. Ceux de Gersaint au XviII ${ }^{e}$ siècle se limitent à un frontispice conçu par Boucher, qui adopte le même registre iconographique que dans les livres de curiosités : des coraux, madrépores et coquilles soigneusement rangés dans un cabinet (Glorieux 2002 : 279-281, 360-362 et passim) (Figure 22).

Les publications deviennent aussi périodiques par le biais des mémoires d'académies puis de revues scientifiques spécifiquement consacrées aux coquillages et mollusques, sans compter les colloques et symposiums, plus récents. Les publications, enfin, deviennent 
électroniques. Les bibliothèques numérisent leurs livres, dont ceux sur les coquilles, et des bases de données leur sont consacrées. Le site de la Biodiversity Heritage Library comprend une section Shells ${ }^{8}$.

Et les illustrations de continuer leur évolution parallèlement aux techniques. Jadis, lors d'expéditions scientifiques, des cartes du pays et de ses rivages s'imposaient, depuis celles du Sénégal publiées par Adanson. Aujourd'hui, ce sont les fonds sous-marins et lieux de prélèvement qui font l'objet de la cartographie, tandis que des cartes des provinces zoogéographiques marines ou terrestres indiquent l'aire de répartition des espèces. Pour les guides destinés aux collectionneurs, les cartes mettent quelquefois l'accent sur les îles et archipels où se trouvent quelques coquillages rares ou abondance de spécimens. Radiographies, photographies en couleurs, photographies au microscope électronique, tableaux, graphiques et schémas sont d'autres illustrations contemporaines nécessaires au propos scientifique ou vulgarisateur, qu'intègre désormais la mise en page.

\section{Peindre le Peigne de Vénus}

Peindre les coquilles demeure encore au $x^{e}$ siècle une rémanence des anciens livres de coquilles, qui s'exprime dans les techniques ou les formats. En témoigne un guide pour collectionneurs, Beautiful Shells of New Zealand, publié en 1908 par E.G.B. Moss [38], illustré de planches de coquilles disposées comme à l'âge baroque par le photographe C. Spencer. En 1915, l'Album consacré au musée Hirase à Kyoto [3] est illustré de photographies des salles d'exposition et de coquilles placées avec goût, le tout dans un format oblong. En 1936, un recueil de Paul A. Robert, intitulé Les Merveilles de la mer, inclut un texte de Paul Valéry [44]. L'illustrateur y utilise la technique de l'aquarelle et non la photographie, et son nom y précède celui de l'auteur du texte, fût-il Valéry, comme du temps de Redouté, dont le nom éclipsait bien souvent celui du botaniste. Un guide pour collectionneurs, Les coquillages marins du monde en couleurs, paraît en 1975 [42]. L'auteur est un collectionneur, A.P.H. Oliver, et l'illustrateur le peintre James Nicholls, qui lui aussi utilise l'aquarelle. Un format carré, proche du tableau, est utilisé en 1969 pour Les coquillages : les chefs-d'œuvre de la vie sous-marine, un livre de Stix et Abbot illustré de photos d'art [51]. Les coquilles y sont placées sur des plaques de verre et sous éclairage particulier. En 2010, enfin, un livre de photos est consacré aux spécimens de la collection Philippe Dautzenberg [6]. Les photos d'Emmanuel Berry sont en belle page, à droite, et le cadrage est serré, centré sur l'ouverture de la coquille, promouvant celle-ci au rang d'œuvre d'art et de sujet littéraire. Voyage intérieur encore, ambiguité du regard et résurgences de pratiques et techniques anciennes. 


\section{Haïkus}

Encore ne s'agit-il que des livres de coquilles relevant de la tradition occidentale. En Extrême-Orient, ils sont d'une autre nature. Consacrés aux bienfaits de la mer, ils associent des haïkus et des illustrations rehaussées de poudre de métal pulvérisée afin de faire briller les couleurs et de simuler l'éclat des coquilles ou des écailles de poisson (Kozyreff 1992, n 11 et 28). Ces recueils ont été très prisés des Japonais, le pays dépendant largement de la mer. Leurs images de style naturaliste traduisent autant l'esthétique des

Katsuma Ryûsui,

Umi no sachi

[Les bienfaits de la mer]. Edo, 1762,

Pinna et truite de mer, gravures en

couleurs rehaussées de poudre de

métal pulvérisée

(Bibliothèque royale de Belgique,

FS XLI 716).

(Fig. 23) coquillages que le fait de les consommer car elles en montrent aussi les parties molles, l'animal lui-même (Figure 23). Parfois, les coquilles sont disposées sur la plage, comme dans les frontispices occidentaux, placées dans des paniers et associées à des crustacés et poissons. Ce « petit corps calcaire creux et spiral » (Valéry 1936) a donc suscité un intérêt universel et parfois des représentations convergentes, la plage constituant partout un lieu de passage en forme de rituel.

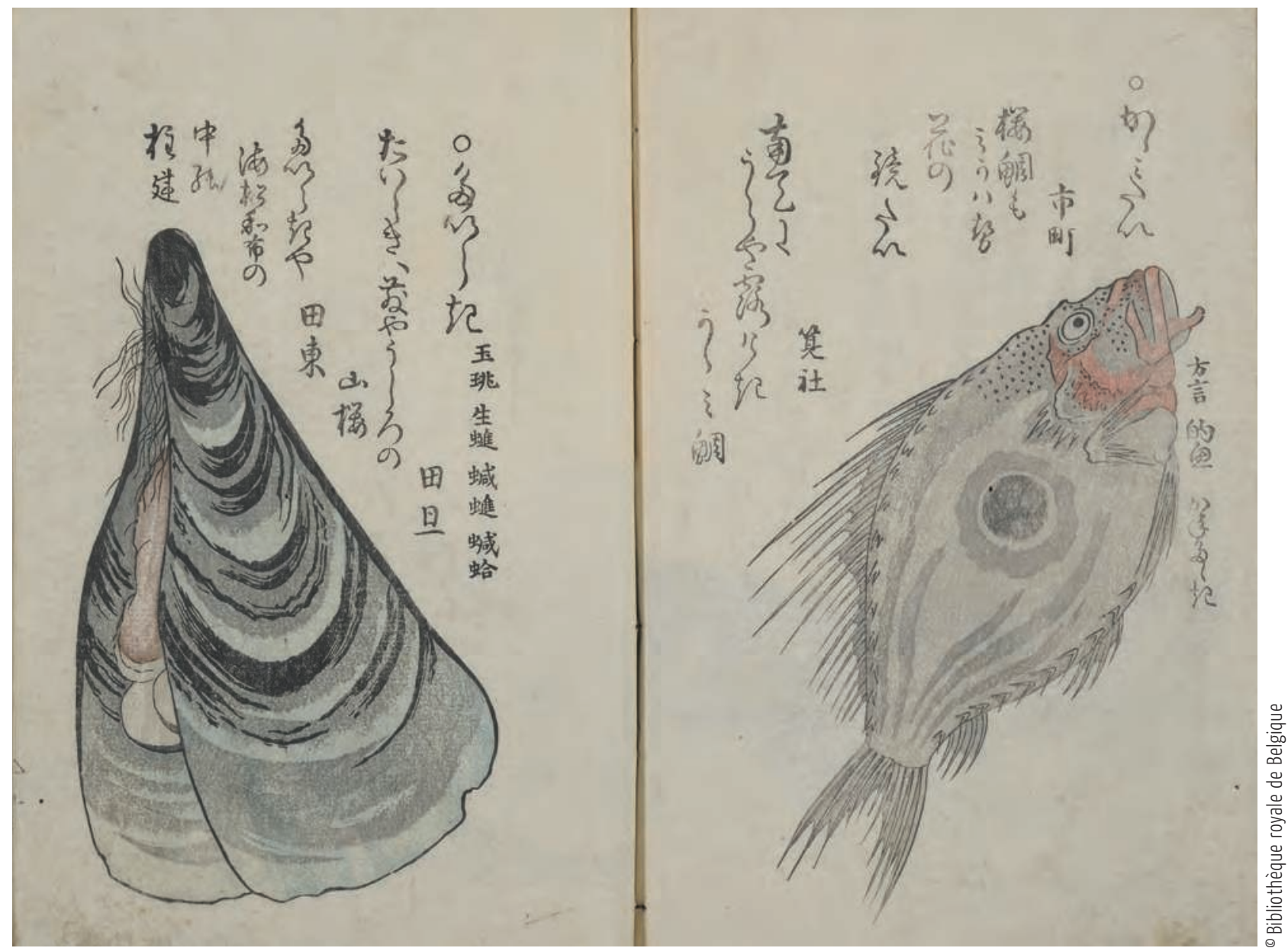




\begin{abstract}
$\&$
L'évolution des livres de coquilles est sensiblement la même que d'autres au sein de l'histoire naturelle. Il ne manque pas de florilèges, de jardins ou de bestiaires, richement illustrés, parallèles à des traités scientifiques, même aujourd'hui. Mais les livres de coquilles sont probablement ceux dans lesquels le sujet même est promu au rang de merveille de la manière la plus éclatante, entretenant une forme d'ambiguité entre curiosité et spécimen, jusqu'à aujourd'hui, tant leur lien avec le monde des cabinets et collections a mis longtemps à se relâcher, sans se dénouer complètement. Même le regard des scientifiques joue sur l'ambiguité, comme Peter Dance qui consacre un livre aux coquillages les plus rares (Dance 1969). Et puis, jadis, il y a ce phénomène unique dans l'illustration qu'est l'incapacité de certains dessinateurs et graveurs à comprendre le sens des coquilles, ce qui donne singularité à ces livres anciens.

Au cours du temps, on se déplace insensiblement des curiosités aux spécimens d'histoire naturelle, du rare au commun, du lointain au proche, des collections privées aux muséums, de l'eau de mer à l'eau douce et à la terre, de la coquille au mollusque, de la conchyliologie à la malacologie, du désordre de la curiosité à l'ordre naturel enfin. Les coquilles se prêtent volontiers au thème du passage dans les livres. Elles voyagent de genre en genre, de livre en livre, parfois au sein des livres en changeant de statut, de place et même de sens. Et le regard de se déplacer lui aussi. Tout en restant émerveillé.
\end{abstract}




\section{NOTES}

Photo d'ouverture : Manuscrit didactique sur les coquilles joint à une copie manuscrite des planches de Burtin, Oryctographie de Bruxelles, XVIII ${ }^{\mathrm{e}}$ siècle (Bibliothèque royale de Belgique, Ms. II 472 B).

1. Les livres anciens cités dans le texte sont repris en références [numérotation dans le texte] dans le catalogue des œuvres citées.

2. Thesaurus conchyliorum, a treasury of books about mollusks and their shells 1999 Catalogue 278 de l'Antiquariaat Junk B.B. à Amsterdam, (introduction de S.P. Dance).

3. Voir : http://www.mathcurve.com/courbes2d/logarithmic/spiraledor.shtml et http://accromath.uqam. ca/contents/pdf/nautile.pdf consultés le 6 mars 2013.

4. Pour l'héraldique, autre exemple de cette difficile transition, voir Pastoureau (1997).
5. Sander Rang [47] (1829), annonce au verso du titre.

6. Testacei cioe nicchi chioccioe e conchiglie. Disegnate e miniate da Jacopo Tosi, 1683, Bologne: Bibliothèque universitaire, Ms. 4312.

7. Bruxelles, Institut royal des Sciences naturelles de Belgique, Bibliothèque Philippe Dautzenberg, 51624, Description très conçise du cabinet de M. l'abbé de Crillon, 1776. Nos remerciements au Prof. Thierry Backeljau, Département des Invertébrés, Institut royal des Sciences naturelles de Belgique.

8. http://www.biodiversitylibrary.org/subject/Shells.

\section{RÉFÉRENCES}

\section{Catalogue des œuvres citées}

[1] Adams, Charles Baker, Catalogue of shells collected at Panama, New York, 1852.

[2] Adanson, Michel, Histoire naturelle du Sénégal. Coquillages, Paris, 1757.

[3] The Album of the Hirase Conchological Museum, Kyoto, 1915.

[4] Belon, Pierre, De aquatilibus libri duo, Paris, 1553.

[5] Bernouilli, Jacques, « Specimen alterum calculi differentialis in dimetienda Spirali Logarithmicae... », Acta eruditorum anno M. DC LXXXXI publicata, Lipse, 1691, p. 282-290.

[6] Berry, Emmanuel, Coques, coquilles, coquillages. 50 photographies. Texte de Marie Guillot, [Bazas], 2010.

[7] Born, Ignaz von, Index rerum naturalium Musei caesarei Vindobonensis. Pars prima. Testacea, Vienne, 1778.

[8] —, Testacea Musei caesarei Vindobonensis, quae iussu Maeriae Theresiae Augustae disposuit et descripsit Ignatius a Born, Vienne, 1780.

[9] Buonanni, Philippo, Ricreatione dell'Occhio e della Mente, Rome, 1681 ; Recreatio mentis et oculi in observatione animalium testaceorum. Rome, 1684.

[10] —, Musaeum Kircherianum, Rome, 1709.

[11] Burmeister, Hermann, Zoologischer Hand-Atlas zum Schulgebrauch und Selbstunterricht, Berlin, 1835.

[12] Burtin, François-Xavier, Oryctographie de Bruxelles ou Description des fossiles tant naturels qu'accidentels découverts jusqu'à ce jour dans les environs de cette ville, Bruxelles, 1784.

[13] Canals y Marti, Juan Pablo, Memorias sobre la purpura de los antiguos... Madrid, 1779.

[14] Ceruto, Benedicto ; Chiocco, Andrea, Musaeum Francesc. Calceolari, Vérone, 1622.

[15] Chenu, Jean-Charles, Illustrations conchyliologiques ou description et figures de toutes les coquilles connues, vivantes et fossiles, Paris, 1842-1853.

[16] Cubières, S.L.P., Histoire abrégée des coquillages de mer : de leurs murs, et de leurs amours, Versailles, an VIII. 
[17] Cuvier, Georges, Mémoires pour servir à l'histoire et à l'anatomie des mollusques. Paris, 1817.

[18] Demidoff, Paul, Catalogue systématique des livres de la bibliothèque de Paul de Demidoff, Moscou, 1806.

[19] Dezallier d'Argenville, Antoine-Joseph, L'Histoire naturelle éclaircie dans deux de ses parties principales, la lithologie et la conchyliologie, dont l'une traite des pierres et l'autre des coquillages, Paris, 1742 ; L'Histoire naturelle éclaircie dans une de ses parties principales, la conchyliologie, Paris, 1757 ; Favanne, Jacques de, Favanne, Jacques-Guillaume de édit., La Conchyliologie ou Histoire naturelle des coquilles de mer, d'eau douce, terrestres et fossiles, avec un traité de la zoomorphose ou représentation des animaux qui les habitent, Paris, 1780.

[20] Ducrotay de Blainville, Henri-Marie, Manuel de malacologie et de conchyliologie, Paris, 1825.

[21] Fichtel, Leopold von ; Moll, Johan Paul Carl von, Testacea microcospica... ex generibus Argonauta et Nautilus... Microscopische und andere Schalthiere aus den Geschlechtern Argonaute und Schiffer, Vienne, 1803.

[22] Gualtieri, Niccoló, Index testarum conchyliorum, Florence, 1742.

[23] Hanley, Sylvanus, Photographic Conchology, Londres, 1863.

[24] —, The Young Conchologist's Book of Species, Londres, 1840.

[25] Hanley, Sylvanus; Theobald, W., Conchologia indica, being illustrations of the land and freshwater shells of British India, Londres, 1870-1876.

[26] Hirase, Yoichiro, Kaisenshu, Kyoto, 1914-1915.

[27] Imperato, Ferrante, Dell'historia naturale libri XXVIII, Naples, 1599.

[28] Issel, Arturo, Malacologia del Mar Rosso, Pise, 1869.

[29] Knorr, Georg Wolfgang, Délices des yeux et de l'esprit, Nuremberg, 1764-1773.

[30] Knorr, Georg Wolfgang; Walch, Johann Ernst Immanuel, Recueil des monumens des catastrophes que le globe a essuiées, Nuremberg, 1777-1778.

[31] Lamy, Édouard, Deuxième expédition antarctique française (1908-1910) commandée par le Dr Jean Charcot. Sciences naturelles : documents scientifiques. Gastropodes, prosopobranches, scaphopode et pélécypodes. Paris, 1911.

[32] Lister, Martin, Exercitatio anatomica In qua de Cochleis terrestribus et limacibus agitur, Londres, 1694.

[33] Lonicerus, Adam, Historiae naturalis, Francfort, 1551-1555.

[34] Martini, Friedrich Wilhelm ; Chemnitz, Johann, Neues systematisches Conchylien-Cabinet, Nuremberg, 1759-1795.

[35] Martyn, Thomas, The Universal Conchologist. Le Conchologiste Universel, Londres, 1784-1787.

[36] Mawe, J., The Voyager's companion, or shell collector's pilot, Londres, 1821.

[37] Melvill, James Cosmo ; Standen, Robert, Catalogue of the Hadfield collection of shells from the Lifu and Uvea, Loyalty Islands, Manchester, 1895-1897.

[38] Moss, E.G.B., Beautiful Shells of New Zealand: An illustrated Work for Amateur Collectors of New Zealand Marine Shells : Photographs by C. Spencer, Auckland, 1908.

[39] Murray, Eustace Clare Grenville, The Oyster; where, how and when to find, breed, cook and it eat, Londres, 1861.

[40] Museum Boltenianum. Verzeichniss der von dem verstorbenen Herrn Joachim Friedrich Bolten... Sammlung Conchylien, Mineralien und Kunstsachen... Hambourg, 1819.

[41] Olearius, Adam, Gottorffische Kunst-Cammer, Schleswig, 1666.

[42] Oliver, A.P.H. ; Nicholls, J. ; The Hamlyn Guide to Seashells of the World, Hamlyn, 1975 ; Idem, Les coquillages marins du monde en couleurs, Paris-Bruxelles,1975.

[43] Rafinesque-Schmaltz, C.S., Principes fondamentaux de somiologie ou les Loix de la nomenclature et de la classification de l'empire organique, Palerme, 1814.

[44] Robert, Paul-A. ; Valéry, Paul, Les Merveilles de la mer. Les coquillages. 15 planches en couleurs d'après les aquarelles de Paul A. Robert. Texte de Paul Valéry. Paris, 1936.

[45] Roux, Polydore, Iconographie conchyliologique ou recueil de planches lithographiées et coloriées : première livraison, Paris-Marseille-Toulon, 1828.

[46] Rumphius, Georg Everhard, D’Amboinssche rariteitkamer, Amsterdam, 1741.

[47] Sander Rang, A., Manuel de l'histoire naturelle des mollusques et de leurs coquilles ayant pour base de classification celle de M. le baron Cuvier, Paris, 1829.

[48] Scheuchzer, Johann Jakob, Physica sacra, Augsbourg-Ulm, 1731 ; Physique sacrée ou Histoire naturelle de la Bible, Amsterdam, 1732. 
[49] Seba, Albertus, Locupletissimi rerum naturalium thesauri accurata descriptio, et iconibus artificiosissimis expressio, per universam physices historiam, Amsterdam, 1734-1765.

[50] Sowerby, G.B. I, A catalogue of the shells contained in the collection of the late Earl of Tankerville, arranged according to the Lamarckian Conchological System, London, 1825.

[51] Stix, Hugh et Marguerite ; Abbot, R. Tucker, Les Coquillages : les chefs-d'œuvre de la vie sous-marine, Paris, 1969, photographies de H. Landshoff.

[52] Thévet, André, La Cosmographie universelle, Paris, 1575.

[53] Tryon, George W., American marine conchology, Philadelphie, 1873.

[54] Valentini, Michael Bernhard, Amphitheatrum zootomicum, Francfort, 1620.

[55] Valentijn, François, Verhandeling der zeehorenkens en zeegewassen in en omtrent Amboina, DordrechtAmsterdam, 1726 et Amsterdam, 1754.

[56] Vincent, Levinus, Wondertooneel der nature, Amsterdam, 1706. 


\section{Bibliographie}

Bassy, A.-M. 1984 Le texte et l'image. In H.-J. Martin, R. Chartier \& J.-P. Vivet (dir.) Histoire de l'édition française. II. Le livre triomphant, 1660-1830. Paris : Fayard/Le Cercle de la librairie : 140-161.

Bergvelt, E., Meijers, Debora J. \& Rijnders, M. (dir.) 2005 Kabinetten, galerijen en musea : het verzamelen en presenteren van naturalia en kunst van 1500 tot heden. Heerlen-Zwolle: Open universiteit Nederland/ Waanders Uitgeverij.

Chatelain, J.-M. 2000 Pour la gloire de Dieu et du roi : le livre de prestige au xvile siècle. In H.-J. Martin (dir.) La naissance du livre moderne (XIV -XVII ${ }^{e}$ siècles) : mise en page et mise en texte du livre français, Paris: Le Cercle de la librairie : 350-363.

Chatelain, J.-M., Pinon, L. 2000 Genres et fonctions de l'illustration au Xvi e siècle. In H.-J. Martin (dir.) La naissance du livre moderne (XIV e-XVII siècles) : mise en page et mise en texte du livre français, Paris : Le Cercle de la librairie : 236-269.

Conihout, I., de. 1996 Les collections encyclopédiques et leurs catalogues. In Roland Schaer (dir.) Tous les savoirs du monde : encyclopédies et bibliothèques de Sumer au XXI siècle, Paris : Flammarion : 278-319.

Coomans, H. E. 1992 Schelpenverzamelingen. In E. Bergvelt, R. Kistemaker (dir.) De wereld binnen handbereik: Nederlandse kunst- en rariteitenverzamelingen 1585-1735, Zwolle : Waanders : 192-203.

Coppens, C., Tavernier, R. 1989 Uit de schelp gekropen : schelpenboeken in de Leuvense Universiteitsbibliotheek, Leuven : KUL. Universiteitsbibliotheek.

Dance, S. P. 1969 Rare shells, Los Angeles : Berkeley.

— 1986 History of Shell Collecting : An Illustrated History, revised edition, Leiden : E. J. Brill.

Deconinck, R. 2010 Au seuil du livre-monument : l'imaginaire architectural du frontispice aux xvI et XVII siècles. In Godelieve Denhaene (éd.), De Renaissance prentkunst in de Zuidelijke Nederlanden. La gravure de la Renaissance dans les Pays-Bas méridionaux, Bruxelles: Archives et Bibliothèques de Belgique, numéro spécial 89 : 139-152.

Driessen-van het Reeve, J. J. 2006 De Kunstkamera van Peter de Grote : de Hollandse inbreng, gereconstrueerd uit brieven van Albert Seba en Johann Damiel Schumacher uit de jaren 1711-1712, Hilversum : Verloren.

Eisenstein, E.L. 1982 Le livre et la culture savante. In H.-J. Martin, R. Chartier \& J.-P. Vivet (dir.) Histoire de l'édition française. I. Le livre conquérant: du Moyen Âge au milieu du xvII siècle. Paris : Fayard/Le Cercle de la librairie : 563-583.

Fumaroli, M. 1994 Sur le seuil des livres : les frontispices gravés des traités d'éloquence (1594-1641). In eidem, L'École du silence : le sentiment des images au XviI siècle, Paris: Flammarion (Idées et recherches) : 325-342.

Glorieux, G. 2002 À l'Enseigne de Gersaint : Edme-François Gersaint, marchand d'art sur le pont Notre-Dame (1694-1750), Seyssel : Champ Vallon.

Heppell, D. 1995 The long dawn of malacology : a brief history of malacology from prehistory to the year 1800, Archives of Natural History: 301-319.

Impey, O., MacGregor, A. (édit.) 1985 The Origins of Museums. The Cabinet of Curiosities in Sixteenth and Seventeenth-Century Europe, Oxford: Clarendon Press.

Jammes, B. 1984 Le livre de science. In H.-J. Martin, R. Chartier \& J.-P. Vivet (dir.) Histoire de l'édition française. II. Le livre triomphant, 1660-1830. Paris : Fayard/Le Cercle de la librairie : 206-217.

Johnson, R. I. 2002 The marvelous, monstrous, mythical, marine mollusk Cochlea sarmatica Thevet, 1575, Department of Mollusks. Harvard University, Occasional Papers on Mollusks, vol. 6 (81) : 149-174.

Kozyreff, C. 1992 Deux siècles de livre japonais : le fonds Hans de Winiwarter, exposition à la Bibliothèque royale Albert I Ir, Bruxelles.

Lamy, É. 1930 Les Cabinets d'histoire naturelle en France au XvIII siècle et le Cabinet du Roi (1635-1793). Rennes : É. Lamy.

Landwehr, J. 1976 Studies in Dutch Books with Coloured Plates published 1662-1875 : natural history, topography and travel, costumes and uniforms, La Haye : Junk. 
Laufer, R. 1989 L'espace visuel du livre ancien. In H.-J. Martin, R. Chartier \& J.-P. Vivet (dir.) Histoire de lédition française. I. Le livre conquérant: du Moyen Âge au milieu du XviI siècle. Paris : Fayard/Le Cercle de la librairie : 479-499.

Lestringant, F., du Crest, S. 2008 Le théâtre de la curiosité. Paris: Presses de l'Université Paris-Sorbonne (Cahiers V. L. Saulnier, 25).

Lugli, A. 1998 Naturalia et Mirabilia. Les cabinets de curiosités en Europe. Paris : Adam Biro.

Martin, P., Moncond'huy, D. 2004 Curiosité et cabinets de curiosités, Neuilly : Atlande.

Marx, J. 1977 Lactivité scientifique de l'Académie impériale et royale des sciences et belles-lettres de Bruxelles. In Bruxelles au XVIII siècle, actes de la journée d'étude 6 novembre 1976. Bruxelles: Études sur le xviII siècle IV : 49-61.

Nissen, C. 1969 Die zoologische Buchillustration : ihre Bibliographie und Geschichte, Stuttgart : Hiersemann.

Pastoureau, M. 1997 La couleur en noir et blanc (xve-XviIl siècle) In F. Barbier et al., Le livre et l'historien: études offertes en l'honneur du Professeur Henri-Jean Martin, Genève : Droz : 197-213

— 1982 L'illustration du livre : comprendre ou rêver. In H.-J. Martin, R. Chartier \& J.-P. Vivet (dir.) Histoire de l'édition française. I. Le livre conquérant: du Moyen Âge au milieu du xvII' siècle, Paris: Fayard/Le Cercle de la librairie, p. 501-529.

Pinault Sørensen, M. 2008 Le livre de botanique XVII et XVIII siècles, Paris : Conférences Léopold Delisle.

Pomian, K. 1987 Collectionneurs, amateurs et curieux. Paris, Venise : XVI ${ }^{e}$-XVIII ${ }^{e}$ siècle, Paris : Gallimard (Bibliothèque des histoires).

Sarmant, T. 2000 Roma triumphans : les frontispices des livres de médailles aux XVII et $\mathrm{XVIII}^{\mathrm{e}}$ siècles. In Textes imprimés et images $X V I^{e}$-XIXe siècles, études réunies par Annie Charon, Isabelle Diu \& Elisabeth Parinet Bibliothèque de l'École des Chartes 158: 69-95.

Schnapper, A. 1988 Le Géant, la licorne et la tulipe : collections et collectionneurs dans la France du XVII siècle. I. Histoire et histoire naturelle, Paris : Flammarion (Art, Histoire, Société).

Sorgeloos, C. 1996 Les cabinets d'histoire naturelle et de physique dans les Pays-Bas autrichiens et à Liège. In M.-Th. Isaac et C. Sorgeloos (dir.) La diffusion du savoir scientifique (XVI'-XXe siècle). Actes du colloque de l'Université de Mons-Hainaut, 22 septembre 1995. Bruxelles : Archives et Bibliothèques de Belgique, numéro spécial $51: 125-230$.

Valéry, P. 1936 L'homme et la coquille. In Les Merveilles de la mer, Paris : Gallimard, 14.

Visser, R. 2005 De ontwikkeling van het natuurhistorische museum sedert 1750. In Ellinoor Bergvelt, Debora J. Meijers, Kabinetten, galerijen en musea : het verzamelen en presenteren van naturalia en kunst van 1500 tot heden. Heerlen : Open Universiteit ; Zwolle : 179-202.

\section{Sites}

Biodiversity Heritage Library, qui reproduit la plupart des livres cités ici : http://www.biodiversitylibrary. org/subject/Shells (consulté le 9 mars 2013)

Pierre Martin et Dominique Moncond'huy, Les cabinets de curiosités et le livre, journée d'étude le 4 décembre 2008, à l'Espace Mendès-France, Poitiers : http://curiositas.org/agenda/les-cabinets-de-curiosites-etle-livre (consulté le 9 mars 2013) 


\section{RÉSUMÉ}

Murex barclayi : livres de coquilles. Les livres de coquilles se déclinent en différents genres, chacun ayant son public, ses formes et ses usages : livres d'histoire naturelle, livres destinés aux collectionneurs et cabinets de curiosité, traités de conchyliologie et de malacologie, publications scientifiques et guides pour collectionneurs. Tous ont des formats et des illustrations correspondant aux publics et lecteurs.

\section{ABSTRACT}

Murex barclayi: books shells. There are different kinds of books representing shells, each of them for a different purpose and another public : treatises of natural history, books for collectors and cabinets of curiosities, treatises of conchyliology and malacology, scientific publications and guides for collectors. All of them have different formats and kinds of illustration, for each reader.

\section{MOTS CLÉS}

Coquillage, conchyliologie, malacologie, histoire du livre, illustration, curiosité, collection, guide

\section{KEYWORDS}

Shell, conchyliology, malacology, book history, illustration, curiosity, collection, guide 